\title{
The Kowalewski Top 99 Years Later: A Lax Pair, Generalizations and Explicit Solutions
}

\author{
A. I. Bobenko, A. G. Reyman, and M. A. Semenov-Tian-Shansky \\ Leningrad Branch of V. A. Steklov Mathematical Institute, Fontanka 27, \\ SU-191011 Leningrad, USSR
}

\begin{abstract}
A "natural" Lax pair for the Kowalewski top is derived by using a general group-theoretic approach. This gives a new insight into the algebraic geometry of the top and leads to its complete solution via finite-band integration theory.
\end{abstract}

\section{Introduction}

In her celebrated paper [1] published in 1889 Kowalewski found a new and highly nontrivial integrable case of the motion of a heavy rigid body about a fixed point, completing the list of integrable tops. Two previously known integrable cases are relatively simple and had been solved already in the XVIII ${ }^{\text {th }}$ century. These are Euler's top in which the stationary point coincides with the center of mass, and Lagrange's top which is axially symmetric. The third case discovered by Kowalewski is rather bizarre: the moments of inertia have a fixed ratio $2: 2: 1$, and the center of mass lies in the equatorial plane of the top. This case was detected by requiring that the general solution be given by meromorphic functions of the complex time variable. Unlike most other integrable systems of mechanics known in the XIX ${ }^{\text {th }}$ century, the Kowalewski top cannot be solved by separation of variables. To integrate it, Kowalewski used an ingeneous change of variables which reduced the problem to hyperelliptic quadratures. However, the inverse change of variables leads to highly complicated expressions in terms of hyperelliptic theta functions which seem completely unmanageable (explicit formulae for the "physical" variables of the top were derived by Kötter [2]).

The new powerful method of finite-band integration created by Novikov, Dubrovin, Matveev, Its, Krichever and others (see [3] for a review) has led to a revival of interest in integrable problems of mechanics. Lax pairs have been found for many classical integrable systems, in particular, for various integrable cases of motion of a rigid body and for their multi-dimensional analogs. The Kowalewski top, however, has long remained a remarkable exception which baffled numerous attempts to build an adequate Lax pair for it. The only Lax pair known to the authors before 1987 was proposed by Perelomov [4]; it did not contain a spectral parameter and so was of no help in solving the equations of motion. 
Recently the last two authors have found a "natural" Lax representation with a spectral parameter for the Kowalewski top [5]. It is based on a general grouptheoretical approach to integrable systems $[6,7]$ and makes use of the Lie algebra so $(3,2)$. We believe that this construction fully explains the peculiar geometry of the Kowalewski top and the origin of its integrability. It turns out that the Kowalewski top is the reduction of a more symmetrical system with extra degrees of freedom, namely, a spherical top interacting with a two-dimensional rotator. As a matter of fact, the construction leads automatically to a new, more general integrable system - the Kowalewski gyrostat in two constant field [see formula (1.5)]. Applying this construction to the Lie algebras $s o(p, q)$ we obtain multidimensional analogs of the Kowalewski top [see (2.4)].

The Lax pair given in [5] and reproduced here also provides a new insight into the algebraic geometry of the Kowalewski top: it leads to an algebraic curve different from the Kowalewski curve as explained in Sect. 5. The new curve has genus 3 and is not hyperelliptic but is a two-sheeted cover of an elliptic curve. The completed complex Liouville torus for the Kowalewski top coincides with the Prym variety of this covering. Using the machinery of finite-band integration theory, the first author [13] obtained concise explicit expressions for the solutions of the Kowalewski top which are much simpler than the original formulae of Kowalewski and Kötter [see formulae (7.42), (7.64), (7.74) below]. Their derivation is natural in the sense that it uses only the standard finite-band integration technique and avoids any artificial change of variables which was so crucial in the computations of Kowalewski. Furthermore, this technique also gives explicit solutions for the Kowalewski gyrostat in two constant fields (we do not present these latter formulae here because of lack of space).

An alternative algebro-geometric approach to the study of the Kowalewski top has been recently developed in [8-10], based on a general method due to Adler and van Moerbeke. It avoids an a priori knowledge of the Lax representation; its starting point, as in the original Kowalewski paper, is the study of the blow-up of solutions in the complex time variable. It was shown that the level surfaces defined by the four constants of the Kowalewski motion complete into Abelian surfaces of polarization (2.4) by adjoining a divisor $\mathscr{D}=\mathscr{D}_{1}+\mathscr{D}_{2}$; the two isomorphic curves $\mathscr{D}_{1}$ and $\mathscr{D}_{2}$ each have genus 3 and are double covers of elliptic curves. The compactified Liouville tori in this approach are identified with the duals of the Prym varieties of any of these coverings. Note that these curves are different from the ones defined in our paper. A beautiful and detailed analysis of the linear systems associated with the divisors $\mathscr{D}, \mathscr{D}_{1}, \mathscr{D}_{2}$ carried out in $[8,9]$ allows to establish a birational isomorphism between the Kowalewski flow on the Liouville tori and the flows of other algebraically integrable systems that are linearizable on abelian varieties of the same type. Specifically, the isomorphism in [9] relates the Kowalewski top to the Manakov top on so(4) and the one in [8] to the Clebsch case of the motion of a rigid body in ideal fluid. Lax pairs for these systems are well known and yield as a byproduct Lax representations for the Kowalewski top. In fact, [9] exhibits a one-parameter family of birational maps and hence a oneparameter family of the induced Lax pairs. However, the expressions for the Lax pairs obtained in this way in terms of the dynamical variables of the top are rather complicated and cannot be extended to the case of the gyrostat in two fields, or to 
the multi-dimensional case. Moreover, the isomorphism established in $[8,9]$ does not respect the Hamiltonian structure of the equations, and the parameters of one system depend on the integrals of motion of the other.

It is also interesting to recall an earlier result of Dubrovin and Novikov [11] who observed that the equations of motion expressed in the Kowalewski variables coincide with the so-called Dubrovin equations for the two-band solutions of the $\mathrm{KdV}$ equation (provided that the associated hyperelliptic curves coincide). Hence the Kowalewski flow is isomorphic up to isogeny to the two-band $\mathrm{KdV}$ flow, but this does not yield a bona fide Lax pair for the Kowalewski top. This realization of the Kowalewski flow enabled Novikov and Veselov [12] to construct action-angle variables for the Kowalewski top.

The present paper is a sequel to [5] and contains a detailed exposition of its results as well as a thorough study of the algebraic geometry of the Kowalewski top by means of the Lax pair and explicit formulae for its solutions announced in $[13,22,23]$. The paper is organized as follows. Sections $1-3$ deal with the geometry of the Lax pair for the generalized Kowalewski system. Section 4 contains generalities on the linearization of the corresponding equations of motion. In Sect. 5 we study the algebraic curves associated with the Kowalewski gyrostat in two fields. Starting with Sect. 6 we confine ourselves to the study of the classical Kowalewski case. In Sect. 6 the analytic properties of the Baker-Akhiezer function are described and a general pattern of its computation is outlined. The computation is carried out in Sect. 7 which culminates in explicit formulae for the solutions of the Euler-Poisson equations (Theorem 7.7) and for the motion of the frame attached to the top (No. 7.6). We also discuss the generalized Dubrovin equations for the divisor of the Baker-Akhiezer function. Finally, in No. 7.9 we describe solutions of the top in the case $(\ell, g)=0$ which requires special treatment.

\section{The Kowalewski Gyrostat in Two Constant Fields}

The rotation of a rigid body about a fixed point in two constant fields (say, gravitational and electric) can be described in the moving frame by the EulerPoisson equations

$$
\begin{aligned}
& \frac{d \ell}{d t}=[\ell, \omega]+\left[c_{1}, g\right]+\left[c_{2}, h\right], \\
& \frac{d g}{d t}=[g, \omega], \quad \frac{d h}{d t}=[h, \omega] .
\end{aligned}
$$

Here $\ell$ is the angular momentum of the body, $g$ and $h$ are the gravitational and electric field vectors, the constant vectors $c_{1}$ and $c_{2}$ indicate the centers of mass and charge, $[$,$] denotes vector product in \mathbb{R}^{3}$, and $\omega$ is the angular velocity related to $\ell$ by

$$
\omega=\mathscr{J} \ell+\kappa,
$$

where $\mathscr{J}$ is a symmetric positive definite matrix $\left(\mathscr{J}^{-1}\right.$ is the inertia tensor), and $\kappa$ is a constant vector, the so-called gyrostatic momentum. 
Equations (1.1) are Hamilton's equations of motion with respect to the following Poisson brackets:

$$
\begin{gathered}
\left\{\ell_{i}, \ell_{j}\right\}=\varepsilon_{i j k} \ell_{k}, \quad\left\{\ell_{i}, g_{j}\right\}=\varepsilon_{i j k} g_{k}, \quad\left\{\ell_{i}, h_{j}\right\}=\varepsilon_{i j k} h_{k}, \\
\left\{g_{i}, g_{j}\right\}=\left\{g_{i}, h_{j}\right\}=\left\{h_{i}, h_{j}\right\}=0,
\end{gathered}
$$

where the components $\ell_{i}, g_{i}, h_{i}, i=1,2,3$, are taken in the orthogonal frame $\left(e_{1}, e_{2}\right.$, $e_{3}$ ) attached to the body. The corresponding Hamiltonian is

$$
H=\frac{1}{2}(\mathscr{J} \ell, \ell)+(\kappa, \ell)-\left(g, c_{1}\right)-\left(h, c_{2}\right),
$$

where $($, ) denotes euclidean scalar product. Dynamical systems of this kind are usually called gyrostats. Notice that $|g|^{2},|h|^{2}$ and $(g, h)$ are trivial integrals of motion (Casimir functions) for the Poisson bracket (1.3).

The Kowalewski gyrostat in two constant fields is described by the Hamiltonian

$$
H=\frac{1}{2}\left(\ell_{1}^{2}+\ell_{2}^{2}+2 \ell_{3}^{2}+2 \gamma \ell_{3}\right)-g_{1}-h_{2},
$$

so that the inertia tensor is $\mathscr{J}^{-1}=\operatorname{diag}(1,1,1 / 2)$. The centers of mass and charge lie on the orthogonal axes $e_{1}$ and $e_{2}$ in the equatorial plane of the inertia ellipsoid, while the gyrostatic momentum $\kappa=\gamma e_{3}$ is parallel to its symmetry axis $e_{3}$. The original Kowalewski case corresponds to $\gamma=0,[g, h]=0$ (we can simply put $h=0$ ). In this case the system is $S O(2)$-symmetric with respect to rotations about the field vector $g$, which is reflected by the additional integral of motion $F_{1}=(\ell, g)$.

There is another case of additional $S O(2)$-symmetry: $|g|=|h|,(g, h)=0$. The symmetry group here consists of simultaneous rotations about the axes $e_{3}$ and $[g, h]$ with Hamiltonian

$$
F_{2}=\ell_{3}-(\ell,[g, h]) /|g|^{2} .
$$

Theorem 1.1. The Kowalewski gyrostat defined by the Hamiltonian (1.5) is completely integrable and admits a Lax representation $d L / d t=[L, M]$ given by

$$
\begin{aligned}
& L(\lambda)=\left(\begin{array}{cccc}
g_{1}-h_{2} & g_{2}+h_{1} & g_{3} & h_{3} \\
g_{2}+h_{1} & -g_{1}+h_{2} & h_{3} & -g_{3} \\
g_{3} & h_{3} & -g_{1}-h_{2} & g_{2}-h_{1} \\
h_{3} & -g_{3} & g_{2}-h_{1} & g_{1}+h_{2}
\end{array}\right) \lambda^{-1} \\
& +\left(\begin{array}{cccc}
0 & -\gamma & -\ell_{2} & -\ell_{1} \\
\gamma & 0 & \ell_{1} & -\ell_{2} \\
\ell_{2} & -\ell_{1} & -2 \lambda & -2 \ell_{3}-\gamma \\
\ell_{1} & \ell_{2} & 2 \ell_{3}+\gamma & 2 \lambda
\end{array}\right) \\
& M(\lambda)=\frac{1}{2}\left(\begin{array}{cccc}
0 & -2 \ell_{3}-\gamma & \ell_{2} & \ell_{1} \\
2 \ell_{3}+\gamma & 0 & -\ell_{1} & \ell_{2} \\
-\ell_{2} & \ell_{1} & 2 \lambda & 2 \ell_{3}+\gamma^{\prime} \\
-\ell_{1} & -\ell_{2} & -2 \ell_{3}-\gamma & -2 \lambda
\end{array}\right) .
\end{aligned}
$$

The invariants of the matrix $L(\lambda)$ are integrals of the motion in involution. 
In particular, we have $H=-1 / 4 \operatorname{Res}\left(\operatorname{tr} L(\lambda)^{2} d \lambda / \lambda\right)-\gamma^{2} / 2$. The coefficients of $\operatorname{tr} L(\lambda)^{4}$ provide two additional integrals of the motion in involution which ensure complete integrability:

$$
\begin{gathered}
I_{1}=(\ell, g)^{2}+(\ell, h)^{2}+2\left(\ell_{3}+\gamma\right)(\ell,[g, h]) \\
+2(g, h)\left(g_{2}+h_{1}\right)-2 g_{1}|h|^{2}-2 h_{2}|g|^{2}, \\
I_{2}=\left(\ell_{1}^{2}-\ell_{2}^{2}+2 g_{1}-2 h_{2}\right)^{2}+4\left(\ell_{1} \ell_{2}+g_{2}+h_{1}\right)^{2} \\
-4 \gamma\left(\left(\ell_{3}+\gamma\right)\left(\ell_{1}^{2}+\ell_{2}^{2}\right)+2 \ell_{1} g_{3}+2 \ell_{2} h_{3}\right) .
\end{gathered}
$$

Observe that if $h=0$ the integral $I_{1}$ reduces to $(\ell, g)^{2}$. In the second case of additional $S O(2)$-symmetry, where $(g, h)=0,|g|=|h|$ (this integrable case was also found in [24]) $I_{1}$ is simply related to the integral $F_{2}(1.6)$ :

$$
I_{1}=|g|^{2}\left(2 H+F_{2}^{2}+2 \gamma F_{2}\right) \text {. }
$$

$I_{2}$ is an extension of the integral found by Kowalewski [1] and was indicated earlier: in [14] for the case $\gamma=0$ and in [15] for $\gamma \neq 0, h=0$ (see also [24]).

The matrices $L(\lambda), M(\lambda)(1.7)-(1.8)$ obey the symmetry relations

$$
\begin{aligned}
& X(-\lambda)=\eta X(\lambda) \eta, \\
& X(\lambda)^{t}=-\eta X(\lambda) \eta,
\end{aligned}
$$

where $\eta=\left(\begin{array}{cc}\sigma_{2} & 0 \\ 0 & \sigma_{2}\end{array}\right)$ and $\sigma_{2}$ is the usual Pauli matrix, $\sigma_{2}=\left(\begin{array}{cc}0 & -i \\ i & 0\end{array}\right)$. The Lax pair (1.7)-(1.8) actually results from the more natural $s o(3,2)$-valued Lax pair, as will be explained in the next section.

Let us finally point out that the Poisson brackets (1.3) are in fact the LiePoisson brackets for the Lie algebra $e(3,2)=s o(3)+\mathbb{R}^{3}+\mathbb{R}^{3}$, the semi-direct sum of $s o(3)$ and the abelian space $\mathbb{R}^{3}+\mathbb{R}^{3}$, where $s o(3)$ acts on each copy of $\mathbb{R}^{3}$ in the usual way. The dual space $e(3,2)^{*}$ is the phase space for Eqs. (1.1) and decomposes into coadjoint orbits. Generic orbits have dimension 6 and are specified by the Casimir functions $|g|^{2},|h|^{2}$ and $(g, h)$. The condition $[g, h]=0$, which singles out the Kowalewski case, determines a family of 4-dimensional orbits; these may be regarded as orbits of the group of euclidean motions.

\section{Multi-Dimensional Generalizations of the Kowalewski Top}

The rotation of a $p$-dimensional rigid body about a fixed point in $q$ different constant fields is described by the generalized Euler-Poisson equations

$$
\begin{aligned}
& \frac{d \ell}{d t}=[\ell, \omega]+\sum_{i=1}^{q} c_{i} \wedge f_{i}, \\
& \frac{d f_{i}}{d t}=-\omega f_{i}, \quad i=1, \ldots, q .
\end{aligned}
$$

Here $\ell \in S O(p)$ is the angular momentum of the body, $f_{i} \in \mathbb{R}^{p}$ are the field strength vectors in the moving frame (Poisson vectors), $c_{i} \in \mathbb{R}^{p}$ are the corresponding 
(constant) center of charge vectors, and $\omega \in \operatorname{so}(p)$ is the angular velocity related to $\ell$ by a linear transformation $\mathscr{J}$ in $s o(p): \omega=\mathscr{J} \ell$.

Equations (2.1) can be written in Hamiltonian form with respect to the following Poisson brackets in the space of variables $\ell=\left(\ell_{i j}\right), f_{n}=\left(f_{k n}\right), n=1, \ldots, q$ :

$$
\begin{gathered}
\left\{\ell_{i j}, \ell_{k n}\right\}=\delta_{i n} \ell_{j k}+\delta_{j k} \ell_{i n}-\delta_{i k} \ell_{j n}-\delta_{j n} \ell_{i k}, \\
\left\{\ell_{i j}, f_{k n}\right\}=\delta_{j k} f_{i n}-\delta_{i k} f_{j n},
\end{gathered}
$$

where the components of $\ell$ and $f_{n}$ are taken in the orthogonal frame $\left(e_{1}, \ldots, e_{p}\right)$ attached to the body. The corresponding Hamiltonian is

$$
H=-\frac{1}{4} \operatorname{tr}(\mathscr{J} \ell \cdot \ell)-\sum_{i=1}^{q}\left(f_{i}, c_{i}\right) .
$$

Note that the phase space for Eqs. (2.1) may be identified with the dual space of the Lie algebra $e(p, q)=s o(p)+\stackrel{q}{\oplus} \mathbb{R}^{p}$, the semi-direct sum of $s o(p)$ and $q$ copies of $\mathbb{R}^{p}$, so that (2.2) is the associated Lie-Poisson bracket. The inner products $\left(f_{i}, f_{j}\right)$ are trivial integrals of motion for the Poisson brackets (2.2) (in general, this is not a complete set, e.g., for $q \leqq p$ there exist $[(p-q) / 2]$ additional trivial integrals).

Let $p \geqq q$. A $p$-dimensional analogue of the Kowalewski top in $q$ constant fields is defined by the Hamiltonian

$$
H=\frac{1}{4}\left\{\sum_{i, j=1}^{p} \ell_{i j}^{2}+\sum_{i, j=1}^{q} \ell_{i j}^{2}+c \sum_{i, j=q+1}^{p} \ell_{i j}^{2}\right\}-\sum_{i=1}^{q}\left(f_{i}, e_{i}\right) .
$$

It describes a partially symmetric top: the kinetic term in (2.4) has symmetry group $S O(q) \times S O(p-q)$, and the centers of different charges lie on the orthogonal axes $e_{1}, \ldots, e_{q}$. The case $q=p$ corresponds to the spherical top; if $q=1,(2.4)$ gives the generalized Lagrange top (the center of mass lies on the symmetry axis). The angular velocity matrix $\omega$ associated with (2.4) is given by

$$
\begin{aligned}
& \omega_{i j}=2 \ell_{i j}, \quad i, j \leqq q, \\
& \omega_{i j}=\ell_{i j}, \quad i \leqq q<j, \\
& \omega_{i j}=(1+c) \ell_{i j}, \quad i, j>q .
\end{aligned}
$$

We shall now exhibit a Lax pair for the generalized Kowalewski top defined by (2.4). Its coefficients belong to the Lie algebra $s o(p, q)$ which consists of $(p+q)$ $\times(p+q)$-matrices $X$ such that

$$
X^{t}=-I_{p, q} X I_{p, q}
$$

where

$$
I_{p, q}=\operatorname{diag}(1, \ldots, 1,-1, \ldots,-1), \quad \operatorname{tr} I_{p, q}=p-q .
$$

Let $F$ be a $p \times q$-matrix with columns $f_{1}, \ldots, f_{q}$, let $E$ be a $p \times q$-matrix with $E_{i j}=\delta_{i j}$, and let $P$ be the orthogonal projection from $\mathbb{R}^{p}$ onto $\mathbb{R}^{q}\left(\mathbb{R}^{q}\right.$ is spanned by $\left.e_{1}, \ldots, e_{q}\right)$.

Theorem 2.1. The Euler-Poisson equations of a multi-dimensional Kowalewski top with Hamiltonian (2.4) admit a Lax representation which in the natural $(p, q)$-block 
notation is given by

$$
\begin{gathered}
L(\lambda)=\left(\begin{array}{cc}
0 & F \\
F^{t} & 0
\end{array}\right) \lambda^{-1}+\left(\begin{array}{cc}
-\ell & 0 \\
0 & P \ell P
\end{array}\right)+\left(\begin{array}{cc}
0 & E \\
E^{t} & 0
\end{array}\right) \lambda, \\
M(\lambda)=\left(\begin{array}{ll}
\omega & 0 \\
0 & 0
\end{array}\right)-\left(\begin{array}{cc}
0 & E \\
E^{t} & 0
\end{array}\right) \lambda .
\end{gathered}
$$

The invariants of $L(\lambda)$ are integrals of motion in involution.

In particular, for $c=0, H=-1 / 4 \operatorname{Res}\left(\operatorname{tr} L(\lambda)^{2} d \lambda / \lambda\right)$. (Note that the invariants of $L(\lambda)$ Poisson commute with $\sum_{i, j=q+1}^{p} \ell_{i j}^{2}$.)

For $q=2$, the Hamiltonian (2.4) may also include an additional linear term $\gamma \ell_{12}$. In this case $\omega_{12}=2 \ell_{12}+\gamma$ and in the $P \ell P$-block of $L(\lambda)$ one must replace $\pm \ell_{12}$ by $\pm\left(\ell_{12}+\gamma\right)$.

The matrices $L(\lambda)$ and $M(\lambda)$ satisfy, besides (2.6), the symmetry relation

$$
X(\hat{\lambda})=-X(-\lambda)^{t},
$$

which shows that they belong to the twisted affine Lie algebra based on $\operatorname{so}(p, q)$.

The three-dimensional Kowalewski gyrostat discussed in Sect. 1 corresponds to $p=3, q=2$, and so is described by a $5 \times 5$ Lax pair with coefficients in $\operatorname{so}(3,2)$. The more convenient $4 \times 4$ Lax pair (1.7)-(1.8) results by using a 4-dimensional spinor representation of $s o(3,2)$ given by the natural inclusion of $s o(3,2)$ into $s o(3,3) \simeq \operatorname{sl}(4, \mathbb{R})$ or, equivalently, the well-known isomorphism $s o(3,2) \simeq \operatorname{sp}(4, \mathbb{R})$.

To conclude the section, let us point out the following relationship between our Lax pair (2.8)-(2.9) and that of [4]. An easy calculation shows that the evolution of the upper left $q \times q$-block $L^{\prime}$ in the constant term of $L(\lambda)^{2}$ is in turn determined by a Lax equation $d / d t L^{\prime}=\left[L^{\prime}, M^{\prime}\right]$, where $L^{\prime}=P\left(l^{2}+F E^{t}+E F^{t}\right) P$ and $M^{\prime}=P \ell P$. For the case $q=p-1$ this is the representation found in [4].

\section{General Construction of Integrable Systems. Proofs}

The generalizations of the Kowalewski top and Lax pairs for those described in the preceding sections result from a general Lie-algebraic construction. The key observation explaining the peculiar geometry of the Kowalewski top relates it to a more symmetric system with extra degrees of freedom which is furnished by the construction outlined below.

Let $G$ be a Lie group and $\sigma$ an involution in $G$ with fixed point subgroup $K$. The phase space of the integrable systems we are going to describe is the cotangent bundle $T^{*} K$ with canonical symplectic structure. The most interesting examples are obtained when $G$ is a real simple group and $\sigma$ a Cartan involution. In that case $K$ is the maximal compact subgroup of $G$. Some of the Hamiltonians that we shall describe are the sum of a left-invariant kinetic energy which is positive and quadratic in the momenta, and a potential energy depending only on the configuration variables. Clearly, such Hamiltonians admit a natural mechanical interpretation. By examining the list of Riemannian symmetric pairs $(G, K)$ we get a number of interesting mechanical systems [7]. For example, $G=S L(n, \mathbb{R})$, 
$K=S O(n)$ yield $n$-dimensional tops in a quadratic potential (and, as a special case, the Euler-Manakov top). The pair $(S O(n, n), S O(n) \times S O(n))$ gives rise to a system of two interacting $n$-dimensional tops. The exceptional Lie group of type $G_{2}$ leads to an exotic family of integrable tops on $S O(4)$. As we shall readily see, the generalized Kowalewski tops are related to the groups $S O(p, q), p>q \geqq 1$.

Let us now state the main theorem. Let $\mathfrak{g}$ be the Lie algebra of $G$. The involution on $\mathfrak{g}$ induced by $\sigma$ will be denoted by the same letter. Let $\mathfrak{g}=\mathfrak{f}+\mathfrak{p}$ be the Cartan decomposition with respect to $\sigma$ (i.e. $\sigma=\mathrm{id}$ on $\mathfrak{f}, \sigma=-\mathrm{id}$ on $p$ ) and let $\mathfrak{g}^{*}=\mathfrak{f}^{*}+\mathfrak{p}^{*}$ be the dual decomposition. The natural action of $K$ on $\mathfrak{g}^{*}$ (which is the restriction to $K$ of the coadjoint action of $G$ ) leaves $\mathfrak{f}^{*}$ and $\mathfrak{p}^{*}$ invariant. Let us identify $T^{*} K$ with $K \times \mathfrak{f}^{*}$ by means of left translations. Fix two arbitrary elements $a, h \in \mathfrak{p}^{*}$ and to each point $(k, \varrho) \in K \times \mathfrak{f}^{*} \simeq T^{*} K$ assign a Lax matrix

$$
L(\lambda)=h \lambda^{-1}+\varrho+\operatorname{Ad}^{*} k^{-1} \cdot a \lambda,
$$

where $\lambda$ is an auxiliary variable (the "spectral parameter"). Let $I\left(\mathrm{~g}^{*}\right)$ be the algebra of $\mathrm{Ad}^{*} G$-invariant polynomials on $\mathfrak{g}^{*}$. For $\varphi \in I\left(\mathfrak{g}^{*}\right)$ we put

$$
\varphi_{\lambda}(k, \varrho)=\varphi(L(\lambda)) \text {. }
$$

Clearly, $\varphi_{\lambda}$ is a Laurent polynomial in $\lambda$ whose coefficients are polynomial functions of $k, \varrho$. It is more convenient to deal with these coefficients rather than with the "generating function" $\varphi_{\lambda}$. We put

$$
\varphi_{n}=\operatorname{Res}\left(\varphi(L(\lambda)) \lambda^{-n-1} d \lambda\right) .
$$

Let $P_{+}, P_{-}$be the projection operators acting on Laurent polynomials which annihilate all terms of nonpositive (respectively strictly positive) degree. For $\varphi \in I\left(\mathfrak{g}^{*}\right)$ let $d \varphi(L) \in \mathfrak{g}$ be its differential evaluated at $L \in \mathfrak{g}^{*}$.

Theorem 3.1. (i) The functions $\varphi_{n}, \varphi \in I\left(\mathrm{~g}^{*}\right), n \in \mathbb{Z}$, are in involution with respect to the canonical Poisson bracket on $T^{*} K$.

(ii) The equations of motion induced by the Hamiltonian $\varphi_{n}$ on $T^{*} K$ give rise to a generalized Lax equation for $L(\lambda)$ :

$$
\frac{d L}{d t}=-\mathrm{ad}_{\mathrm{g}}^{*} M_{ \pm} \cdot L, \quad M_{ \pm}= \pm P_{ \pm}\left(\lambda^{-n} d \varphi(L(\lambda)) .\right.
$$

Note that ad* $M_{+} \cdot L=\operatorname{ad}^{*} M_{-} \cdot L$, since $\operatorname{ad}^{*} d \varphi(L) \cdot L=0$. In the applications we have in mind $(G, K)$ is a Riemannian symmetric pair. We shall always identify $\mathfrak{g}^{*}$ and $\mathfrak{g}$ by means of an invariant inner product which is positive definite on $f$. In that case (3.4) becomes an ordinary Lax equation

$$
\frac{d L}{d t}=\left[L, M_{ \pm}\right]
$$

We refer the reader to $[7,16]$ for a complete proof of this theorem which uses the twisted loop algebra associated with $(\mathfrak{g}, \sigma)$ and the notion of classical $r$-matrices. Here we present an elementary proof of the first part of the theorem.

The proof proceeds in two steps. Let us observe first that the set of Lax matrices of the form

$$
L(\lambda)=h \lambda^{-1}+\varrho+v \lambda
$$


with $h \in \mathfrak{p}^{*}$ fixed, $\varrho \in \mathfrak{l}^{*}, v \in \mathfrak{p}^{*}$, admits a Poisson structure which agrees with the canonical Poisson structure on $T^{*} K$. To describe this structure we shall start with a slightly more general situation.

Let $\pi$ be a linear representation of $K$ in a vector space $V$. Let $\pi^{*}$ be the dual representation acting in $V^{*}$. Let $\mathfrak{f}+V$ be the semidirect sum of $\mathfrak{f}$ and $V$ (regarded as a $\mathfrak{f}$-module). The dual space $(\mathfrak{f}+V)^{*} \simeq \mathfrak{f}^{*}+V^{*}$ is equipped with the corresponding Lie-Poisson bracket.

Proposition 3.2. Fix an element $a \in V^{*}$. The mapping

$$
\mu_{a}: T^{*} K \rightarrow \mathfrak{f}^{*}+V^{*}:(k, \varrho) \mapsto \varrho+\pi^{*}\left(k^{-1}\right) a
$$

preserves the Poisson brackets, i.e., maps the canonical Poisson bracket on $T^{*} K$ into the Lie-Poisson bracket on $(\mathfrak{f}+V)^{*}$.

Proof. From the definition of the Lie-Poisson bracket it is clear that we must check the equality

$$
[X, Y] \circ \mu_{a}=\left\{X \circ \mu_{a}, Y \circ \mu_{a}\right\}
$$

for arbitrary linear functions $X, Y$ on $(\mathfrak{f}+V)^{*}$. The bracket on the left is the Lie bracket in $\mathfrak{f}+V$. The cases when $X, Y$ belong to $\mathfrak{k}$ or $V$ are treated separately. If $X, Y \in V$, both sides of (3.7) are zero. Let $X \in \mathfrak{f}$. The flow generated by $X$ on $(\mathfrak{f}+V)^{*}$ is given by the action of the 1-parameter subgroup $\exp (-t X)$. The flow of $X \circ \mu_{a}$ on $T^{*} K$ is given by right multiplication by $\exp (t X)$. Clearly, $\mu_{a}$ maps the latter flow onto the former, whence $\left\{X \circ \mu_{a}, \varphi \circ \mu_{a}\right\}=\{X, \varphi\} \circ \mu_{a}$ for any function $\varphi$ on $(\mathfrak{f}+V)^{*}$.

In our special case we have $V=\mathfrak{p}$ and $\pi$ is the natural representation of $K$ in $p$.

Part (i) of Theorem 3.1 is now reduced to the following assertion.

Proposition 3.3. Functions of the form $\varphi_{\lambda}(\varrho, v)=\varphi(L(\lambda)), \varphi \in I\left(\mathrm{~g}^{*}\right)$, are in involution with respect to the Lie-Poisson bracket on $(\mathfrak{l}+\mathfrak{p})^{*}$.

Proof $([17])$. Let us denote the Lie-Poisson bracket on $(\mathfrak{f}+\mathfrak{p})^{*}$ by $\{,\}_{\sigma}$. We shall need two more Poisson brackets defined on the same linear space $(\mathfrak{f}+\mathfrak{p})^{*} \simeq \mathfrak{g}^{*}$, namely, the Lie-Poisson bracket of $g($ denoted by $\{\}$,$) and the bracket \{,\}_{h}$ given by

$$
\{X, Y\}_{h}=h([X, Y]), X, Y \in \mathfrak{g} .
$$

Consider a linear transformation $T_{\lambda}$ on $\mathrm{g}^{*}$ defined by $T_{\lambda}(\varrho+v)=h \lambda^{-1}+\varrho+v \lambda$, $\varrho \in \mathfrak{I}^{*}, v \in \mathfrak{p}^{*}$. It is easily checked that $T_{\lambda}$ carries the bracket $\{$,$\} into$

$$
\{,\}_{\lambda}=\lambda^{-2}\{,\}+\left(1-\lambda^{-2}\right)\{,\}_{\sigma}+\lambda^{-2}\{,\}_{h} .
$$

An invariant function $\varphi \in I\left(\mathrm{~g}^{*}\right)$ is a Casimir function with respect to the bracket $\{$,$\} , i.e. \{\varphi, \psi\}=0$ for any $\psi$. Hence $\varphi_{\lambda}=\varphi \circ T_{\lambda}$ is a Casimir function with respect to $\{,\}_{\lambda}$. If $\psi$ is another ad* $\mathrm{g}$-invariant function, we get $\left\{\varphi_{\lambda}, \psi_{\mu}\right\}_{\lambda}=0=\left\{\varphi_{\lambda}, \psi_{\mu}\right\}_{\mu}$. Therefore, $\varphi_{\lambda}$ and $\psi_{\mu}$ Poisson commute with respect to the bracket $\lambda^{2}\{,\}_{\lambda}$ $-\mu^{2}\{,\}_{\mu}=\left(\lambda^{2}-\mu^{2}\right)\{,\}_{\sigma}$, i.e. $\left\{\varphi_{\lambda}, \psi_{\mu}\right\}_{\sigma}=0$ for $\lambda \neq \mu$, and hence for all $\lambda, \mu$.

It can be shown, more generally, that the $\varphi_{\lambda}$ 's are in involution with respect to a linear family of Poisson brackets $\alpha\{,\}_{\sigma}+\beta\left(\{\}+,\{,\}_{h}\right)$. 
We refer the reader to [7] for an explicit description of all Hamiltonians of the form (3.3) which are quadratic in the momenta. For our present purposes we shall need only the simplest of them.

Before applying Theorem 3.1 to concrete systems it is convenient to make a canonical change of variables $(k, \varrho) \mapsto\left(k^{-1},-\mathrm{Ad}^{*} k \cdot \varrho\right)$ on $T^{*} K$. In the new variables the Lax matrix (3.1) takes the form

$$
L(\lambda)=h \lambda^{-1}-\mathrm{Ad}^{*} k \cdot \varrho+\mathrm{Ad}^{*} k \cdot a \lambda .
$$

Invariant functions on $T^{*} K$ of the form (3.2) have the following obvious symmetries. Let $K_{v} \subset K$ be the isotropy subgroup of an element $v \in \mathfrak{p}$. Then the Hamiltonians $\varphi(L(\lambda)), \varphi \in I\left(\mathfrak{g}^{*}\right)$, are right $K_{a}$-invariant and left $K_{h}$-invariant. Moreover, $L(\lambda)$ does not change under the action of $K_{a}$ by right translation. Hence $L(\lambda)$ effectively describes the system reduced with respect to the action of $K_{a}$. These observations will be important in the study of Kowalewski's top.

As we have already mentioned, the generalized Kowalewski top is connected with the Lie algebra $s o(p, q)$. Recall that by definition it consists of all $(p+q)$ $\times(p+q)$-matrices satisfying

$$
X^{t}=-I_{p, q} X I_{p, q}
$$

where $I_{p, q}=\operatorname{diag}(1, \ldots, 1,-1, \ldots,-1), \operatorname{tr} I_{p, q}=p-q$. In the obvious $(p, q)$-block notation an element $X \in S o(p, q)$ has the form

$$
X=\left(\begin{array}{cc}
\ell & s \\
s^{t} & m
\end{array}\right),
$$

where $\ell=-\ell^{t}, m=-m^{t}$, and $s$ is an arbitrary $p \times q$-matrix. The Cartan involution $\sigma$ is given by $\sigma(X)=-X^{t}$. The maximal compact subalgebra $\mathfrak{f}=s o(p) \oplus \operatorname{so}(q)$ consists of matrices $X$ with $s=0$. The subspace $p$ consists of matrices $X$ with $\ell=0$, $m=0$. The split rank of $s o(p, q)(p \geqq q)$ is clearly equal to $q$. We shall identify $s o(p, q)^{*}$ and $s o(p, q)$ by means of the invariant inner product

$$
(X, Y)=-\operatorname{tr} X Y,
$$

which is positive definite on $f$. The space $I\left(\mathfrak{g}^{*}\right)$ consists of spectral invariants of matrices and is generated by the functions $\varphi_{s}(X)=\operatorname{tr} X^{s}, s \geqq 2$.

Let $k \in S O(p), r \in S O(q), \ell \in S O(p), m \in S O(q)$ be the position and momentum variables on $T^{*}(S O(p) \times S O(q))$. By specializing Theorem 3.1 to the present situation we get the following result.

Theorem 3.4. Let $A, H$ be some fixed $p \times q$-matrices. The spectral invariants of the Lax matrix

$$
L(\lambda)=\left(\begin{array}{cc}
0 & H \\
H^{t} & 0
\end{array}\right) \lambda^{-1}-\left(\begin{array}{cc}
k \ell k^{t} & 0 \\
0 & r m r^{t}
\end{array}\right)+\left(\begin{array}{cc}
0 & k A r^{t} \\
r A^{t} k^{t} & 0
\end{array}\right) \lambda
$$

are in involution with respect to the canonical Poisson bracket on $T^{*}(S O(p) \times S O(q))$. The associated Hamiltonian equations give rise to Lax equations with the Lax matrix (3.12).

For example, the Hamiltonian

$$
H=-\frac{1}{4} \operatorname{Res}\left(\operatorname{tr} L(\lambda)^{2} d \lambda / \lambda\right)=-\frac{1}{4} \operatorname{tr}\left(\ell^{2}+m^{2}\right)-\operatorname{tr}\left(k A r^{t} H^{t}\right)
$$


describes two interacting spherical tops of dimensions $p$ and $q$ and gives rise to a Lax equation $d L / d t=[L, M]$ with

$$
M(\lambda)=-\left(\begin{array}{cc}
0 & k A r^{t} \\
r A^{t} k^{t} & 0
\end{array}\right) \lambda .
$$

The generalized Kowalewski top may be obtained from (3.13) with a special choice of $A$. Recall that the Lax matrix (3.12) actually describes a reduced system since it is invariant with respect to the right action of $K_{a}$. In our case the subgroup $K_{a}$ consists of matrices $\left(\begin{array}{ll}k & 0 \\ 0 & r\end{array}\right)$ such that $k A r^{t}=A$. Now, let $A$ be the truncated identity matrix, $A=E, E_{i j}=\delta_{i j}$. Then $k A r^{t}=A$ implies that $k$ is a block matrix

$$
k=\left(\begin{array}{ll}
r & 0 \\
0 & v
\end{array}\right),
$$

where the lower $(p-q) \times(p-q)$-block is an arbitrary orthogonal matrix. Hence $K_{a}=S O(q) \times S O(p-q)$. Note that the subgroup $S O(q) \subset K_{a}$ is embedded into $K=S O(p) \times S O(q)$ diagonally:

$$
r \mapsto\left(\begin{array}{ccc}
r & 0 & 0 \\
0 & 1 & 0 \\
0 & 0 & r
\end{array}\right)
$$

Let us consider a partial reduction of $T^{*} K$ with respect to the action of this diagonal subgroup. The corresponding moment mapping $\mu: T^{*} K \rightarrow \operatorname{so}(q)^{*}$ is given by

$$
\mu(k, r, \ell, m)=P \ell P+m,
$$

where $P$ is the projection operator from $\mathbb{R}^{p}$ onto the linear span of $\left\{e_{1}, \ldots, e_{q}\right\}$. Reduction over zero amounts to imposing the constraints

$$
P \ell P+m=0, \quad r=1
$$

(the latter is a natural subsidiary condition). The reduced phase space is naturally diffeomorphic to $T^{*} S O(p)$ with its canonical Poisson bracket. Inserting the constraints (3.18) into (3.13) we get the reduced Hamiltonian

$$
H=\frac{1}{4}\left(\sum_{i, j=1}^{p} \ell_{i j}^{2}+\sum_{i, j=1}^{q} \ell_{i j}^{2}\right)-\sum_{i=1}^{q}\left(k e_{i}, h_{l}\right),
$$

which is the generalized Kowalewski Hamiltonian in the stationary frame with $c=0$. Note that to ensure the left invariance of the reduced kinetic energy we need the bi-invariance of the kinetic energy in (3.13). This explains the choice of the Hamiltonian (3.13) which might seem arbitrary.

From (3.12), (3.14) we derive a Lax pair for the reduced system

$$
\begin{gathered}
L(\lambda)=\left(\begin{array}{cc}
0 & H \\
H^{t} & 0
\end{array}\right) \lambda^{-1}-\left(\begin{array}{cc}
k \ell k^{t} & 0 \\
0 & -P \ell P
\end{array}\right)+\left(\begin{array}{cc}
0 & k E \\
E^{t} k^{t} & 0
\end{array}\right) \lambda, \\
M(\lambda)=-\left(\begin{array}{cc}
0 & k E \\
E^{t} k^{t} & 0
\end{array}\right) \lambda .
\end{gathered}
$$


The reduced system still possesses an $S O(p-q)$-symmetry inherited from the isotropy subgroup $K_{a}=S O(q) \times S O(p-q)$. In particular, the spectral invariants of $L(\lambda)$ are in involution with the generators $\ell_{i j}$ for $i, j>q$. Obviously, adding an $s o(p-q)$-Casimir function $H_{1}=c \sum_{i, j>q} \ell_{i j}^{2}$ to (3.19) does not damage the integrability of the system (this term may also be obtained by choosing a suitable invariant of $L(\lambda)$, see [7]). Moreover, the trajectories of $H+H_{1}$ are easily determined once we have found the trajectories of $H$. Indeed, let $\gamma: T^{*} S O(p) \times \mathbb{R}$ $\rightarrow T^{*} S O(p)$ be the flow of $H$ and $\gamma_{1}$ the flow of $H_{1}$. Since $\left\{H, H_{1}\right\}=0$, the flow of $H+H_{1}$ is $\gamma_{1}(\gamma(x, t), t)$.

Lemma 3.5 [18]. Let $\mu: \mathscr{M} \rightarrow \mathrm{g}^{*}$ be the moment map for a Hamiltonian action of a Lie group $G$ on a symplectic manifold $\mathscr{M}$, and let $\Phi$ be an invariant polynomial on $\mathrm{g}^{*}$. Then the trajectories of the collective Hamiltonian $\Phi \circ \mu$ on $\mathscr{M}$ are given by $\exp t X \cdot m$, where $X=d \Phi(\mu(m)), m \in \mathscr{M}$.

In the case we discuss $G=S O(p-q)$ and $\Phi=H_{1}$, so that the flow $\gamma_{1}$ is given by

$$
\gamma_{1}(k, \ell ; t)=\left(k e^{c t \tilde{\ell}}, \operatorname{Ad} e^{-c t \tilde{\ell}} \cdot \ell\right) \text {, }
$$

where $\tilde{\ell}=P \ell P$.

The invariants of $L(\lambda)$ do not form in general a complete set of integrals of motion for the generalized Kowalewski top (indeed, they Poisson commute with the momenta $\ell_{i j}$ for $\left.i, j>q\right)$. One can prove, however, that complete integrability holds modulo the symmetry group $S O(p-q)$ : a complete set is obtained by adding to the spectral invariants of (3.20) a complete involutive set of functions of the generators $\ell_{i j}, i . j>q$.

We also observe that if, for $q=2$, we reduce over a non-zero point of $s o(2)^{*}$ i.e. replace the constraint (3.18) by

$$
P \ell P+m=\gamma e_{1} \wedge e_{2},
$$

we obtain an extra linear "gyrostatic" term $\gamma \ell_{12}$ in the reduced Hamiltonian (3.19). The reduced phase space remains the same. In this case $\pm \ell_{12}$ in the lower $q \times q$ block of the Lax matrix (3.21) is replaced by $\pm\left(\ell_{12}+\gamma\right)$.

We have discussed so far the generalized Kowalewski top in the stationary reference frame. To recover the Lax pair (2.8)-(2.9) we have to go over to the moving frame which amounts to the gauge transformation

$$
L \mapsto \hat{k}^{t} L k, \quad M \mapsto \hat{k}^{t} M \hat{k}+\omega
$$

with $\hat{k}=\left(\begin{array}{ll}k & 0 \\ 0 & 1\end{array}\right), k \in S O(p), \omega=\left(\begin{array}{cc}k^{t} d k / d t & 0 \\ 0 & 0\end{array}\right)$. The matrix coefficients of the transformed matrices may be expressed through the variables

$$
\ell \in \operatorname{so}(p), \quad F=k^{t} H=\left(f_{1}, \ldots, f_{q}\right),
$$

which describe the motion of the top in the Euler-Poisson picture. Note that the angular velocity $\omega$ is expressed through $\ell$ by (2.5). To finish the proof of Theorem 2.1 we need the following lemma which is a special case of Proposition 3.2. 
Lemma 3.6. The mapping

$$
\mu_{H}: T^{*} S O(p) \rightarrow e(p, q)^{*}:(k, \ell) \rightarrow\left(\ell, \hat{k}^{t} H\right)
$$

preserves the Poisson brackets.

Remark. The reader might wonder why we have not used the Euler-Poisson description from the very beginning. To explain the roundabout way taken here recall that the key ingredient of our argument was the involutivity of invariants with respect to the Lie-Poisson bracket of the semi-direct sum $\mathfrak{f}+\mathfrak{p}$ (Proposition 3.3). Now, the mapping from $T^{*} S O(p)$ to $\mathfrak{f}^{*}+\mathfrak{p}^{*}$ associated with the Lax matrix (3.20)

$$
(k, \ell) \mapsto-\left(\begin{array}{cc}
k \ell k^{t} & 0 \\
0 & -P \ell P
\end{array}\right)+\left(\begin{array}{cc}
0 & k E \\
E k^{t} & 0
\end{array}\right)
$$

is a Poisson mapping (note that this fact combined with Proposition 3.3 gives a direct proof of the involutivity of the invariants of the Lax matrix (3.20) without recourse to Hamiltonian reduction). On the other hand, the mapping from $e(p, q)^{*}$ to $(\mathfrak{f}+\mathfrak{p})^{*}$ associated with the Lax matrix (2.8),

$$
\left(\ell, f_{1}, \ldots, f_{q}\right) \mapsto\left(\begin{array}{cc}
-\ell & 0 \\
0 & P \ell P
\end{array}\right)+\left(\begin{array}{cc}
0 & F \\
F^{t} & 0
\end{array}\right)
$$

does not preserve Poisson brackets.

\section{Linearization of the Generalized Kowalewski Flows}

Starting from this section we shall consider complexified equations of motion. It is well known that Lax equations with a spectral parameter are linearizable on the Jacobian of the spectral curve defined by the equation

$$
\operatorname{det}(L(\lambda)-\mu)=0 \text {. }
$$

More precisely, assume that the curve (4.1) is nonsingular and irreducible. Let $\Gamma$ be its nonsingular compactification. Then the eigenspaces of $L(\lambda)$ combine into a holomorphic line bundle $E_{L} \rightarrow \Gamma$. If the matrix $L(\lambda)$ evolves according to a Lax equation, the spectral curve remains invariant and the evolution of $E_{L(t)}$ may easily be determined. We shall analyze this evolution for Lax equations of the form (3.4) described by Theorem 3.1 .

Consider a covering of $\Gamma$ by two open sets $U_{+}=\{p \in \Gamma ; \lambda(p) \neq \infty\}, U_{-}=\{p \in \Gamma$; $\lambda(p) \neq 0\}$. Let $X=\lambda^{-n-1} d \varphi(L(\lambda))$ be the differential of the Hamiltonian (3.3). Since $[L, X]=0$, the eigenvectors of $L(\lambda)$ are also eigenvectors for $X(\lambda)$, hence for an eigenvector $\psi_{p} \in E_{L}(p)$ we have

$$
X(\lambda(p)) \psi_{p}=v(p) \psi_{p} .
$$

Obviously, $v(p)$ is a meromorphic function on $\Gamma$ which is regular in $U_{+} \cap U_{-}$. Let $F_{t}$ be the one-parameter group of line bundles on $\Gamma$ defined by the transition function $\exp t v$ with respect to the covering $\left\{U_{+}, U_{-}\right\}$. 
Theorem 4.1. Suppose $L(t)$ is a solution of the Lax equation (3.4) with the Hamiltonian (3.3). Then the evolution of $E_{L(t)}$ is linear:

$$
E_{L(t)}=E_{L(0)} \otimes F_{t} .
$$

For the proof see $[6,16,17]$.

By the classical Jacobi theorem the group of holomorphic line bundles of degree zero is isomorphic to the Jacobian of $\Gamma$ denoted by $\mathrm{Jac} \Gamma$. Theorem 4.1 shows that the flow on $\mathrm{Jac} \Gamma$ which corresponds to a Lax equation is linear. For its velocity vector we have the following expression. Recall that the tangent space to $\mathrm{Jac} \Gamma$ is dual to the space $\Omega(\Gamma)$ of abelian differentials. If $\omega \in \Omega(\Gamma)$, then the $\omega$-coordinate of the velocity vector $V$ is

$$
\omega(V)=\sum_{p: \lambda(p)=\infty} \operatorname{Res}_{p}(v(p) \omega) .
$$

To solve the Lax equation we use the standard algebro-geometric technique which allows us to reconstruct the Lax matrix from the algebraic data. The set of algebraic (spectral) data consists of the spectral curve $\Gamma$, the line bundle $E_{L}$ and the velocity vector $V$ on $\mathrm{Jac} \Gamma$. It is usually more convenient to work with the dual bundle $E_{L}^{*}$. One can show that under some regularity conditions the degree of $E_{L}^{*}$ is given by $d=g+n-1$, where $g$ is the genus of $\Gamma$ and $n$ is the dimension of the Lax matrix. By the Riemann-Roch theorem $E_{L(t)}^{*}$ has at least $n$ holomorphic sections. Analytically such sections may be regarded as meromorphic functions on $U_{+} \subset \Gamma$ such that (i) $\psi e^{-t v}$ is meromorphic on $U_{-} C \Gamma$ (ii) $\psi$ is subordinate to a (constant) divisor of degree $d$ associated with $E^{*}$. For generic divisors and almost all $t \in \mathbb{C}$ the number of independent sections is precisely $n$. A basis $\psi=\left(\psi_{1}, \ldots, \psi_{n}\right)$ in the space $\mathscr{L}\left(E_{L(t)}^{*}\right)$ is called a vector Baker-Akhiezer function. The functions $\psi_{1}, \ldots, \psi_{n}$ are determined from the algebraic data uniquely up to a linear transformation $\psi \mapsto A \psi, A \in G L(n)$, and satisfy the following basic relations:

$$
\begin{aligned}
& L(\lambda(p)) \psi(p)=\mu(p) \psi(p), \\
& \frac{d \psi(p)}{d t}=-M(\lambda(p)) \psi(p),
\end{aligned}
$$

which serve to reconstruct the Lax matrices. Since $M(\lambda)$ is determined by $L(\lambda)[\mathrm{cf}$. (3.4)] the only freedom in this reconstruction amounts to conjugations $L \mapsto A L A^{-1}, M \mapsto A M A^{-1}$ by a matrix $A$ which does not depend on $\lambda$ and $t$. This freedom may be further reduced by a priori restrictions on the form of the Lax matrices.

Let us now turn to the generalized Kowalewski systems. The Lax pairs (2.8)-(2.9) and (3.20)-(3.21) give rise to the same set of algebro-geometric data, since they differ by a $\lambda$-independent gauge transformation $k(t)$. [Notice that if $p+q$ is odd the spectral curve corresponding to the standard representation of $s o(p, q)$ is reducible and contains a rational component associated with the zero eigenvalue of $L(\lambda)$. This is the main reason why the spinor representation (1.7)-(1.8) for the 3 -dimensional top is more convenient]. Recall now the two additional symmetries (1.12), (1.13) or (2.6), (2.10) of the Lax pair which we write as

$$
\begin{aligned}
& L(\lambda)=I L(-\lambda) I, \\
& L(\lambda)=-I L(\lambda)^{t} I,
\end{aligned}
$$


where $I=I_{p, q}$ for the Lax pair (2.8)-(2.9) and $I=\eta$ for (1.7), (1.8). These symmetries give rise to two commuting involutions $\tau_{1}, \tau_{2}$ on $\Gamma$

$$
\tau_{1}(\lambda, \mu)=(-\lambda, \mu), \quad \tau_{2}(\lambda, \mu)=(\lambda,-\mu) .
$$

The involution $\tau_{1}$ can be lifted to the line bundle $E_{L}$ by setting

$$
\tau_{1} \psi(\lambda, \mu)=I \psi(-\lambda, \mu) .
$$

Let $\tau_{1}^{*}, \tau_{2}^{*}$ be the induced involutions on $\operatorname{Jac} \Gamma$.

Lemma 4.5. The velocity vector of the generalized Kowalewski flow on $\operatorname{Jac} \Gamma$ is invariant under $\tau_{1}^{*}$ and changes sign under $\tau_{2}^{*}$ :

$$
\tau_{1}^{*} V=V, \quad \tau_{2}^{*} V=-V .
$$

Proof. The Hamiltonian of the Kowalewski system is given by $H=-1 / 4 \operatorname{Res}\left(\operatorname{tr} L(\lambda)^{2} d \lambda / \lambda\right)$. Hence its gradient is $1 / 2 L(\lambda)$. Therefore the transition function in Theorem 4.1 is $\exp \mu / 2$ and our claim follows from (4.3).

It is therefore natural to consider the quotient curves $C=\Gamma / \tau_{1}, E=\Gamma /\left(\tau_{1}, \tau_{2}\right)$. Lemma 4.5 and relation (4.8) show that the Kowalewski flow is confined to the subtorus $\operatorname{Jac} C \subset \operatorname{Jac} \Gamma$ and is parallel to the Prym variety of the covering $C \rightarrow E$.

To conclude our discussion of the generalized Kowalewski tops we give the expression for the corresponding velocity vector on $\operatorname{Jac} \Gamma$ (also valid on $\operatorname{Jac} C$ ), which is a specialization of (4.4):

$$
\omega(V)=\sum_{p: \lambda(p)=\infty} \operatorname{Res}_{p} \frac{1}{2} \mu \omega .
$$

We shall now turn to the 3-dimensional case.

\section{The Spectral Curve for the Kowalewski Top}

The characteristic Eq. (4.1) for the Lax matrix (1.7) of the 3-dimensional Kowalewski gyrostat takes the form

$$
\mu^{4}-2 d_{1}\left(\lambda^{2}\right) \mu^{2}+d_{2}\left(\lambda^{2}\right)=0
$$

where

$$
\begin{aligned}
d_{1}(z)= & \left(|g|^{2}+|h|^{2}\right) z^{-1}-\left(2 H+\gamma^{2}\right)+2 z, \\
d_{2}(z)= & \left(\left(|g|^{2}-|h|^{2}\right)^{2}+4(g, h)^{2}\right) z^{-2} \\
& +4\left(I_{1}-\left(H+\gamma^{2} / 2\right)\left(|g|^{2}+|h|^{2}\right)\right) z^{-1} \\
& +\left(I_{2}+4 \gamma^{2} H+\gamma^{4}\right)-4 \gamma^{2} z .
\end{aligned}
$$

The coverings $\Gamma \rightarrow C$ and $C \rightarrow E$ are given by a change of variables $z=\lambda^{2}$ and $y=\mu^{2}$ (this is of course true in the general case), so that the curves $C$ and $E$ are defined by the equations

$$
\mu^{4}-2 d_{1}(z) \mu^{2}+d_{2}(z)=0
$$

and

$$
y^{2}-2 d_{1}(z) y+d_{2}(z)=0 .
$$


The genera of typical curves depending on the values of the Casimir functions are given in Table 1 below $^{1}$

Table 1

\begin{tabular}{|c|c|c|c|c|c|c|c|c|}
\hline & \multirow{3}{*}{$\begin{array}{l}\text { general } \\
\text { case }\end{array}$} & \multirow[t]{3}{*}{$\gamma=0$} & \multicolumn{2}{|c|}{$|g|=|h|,(g, h)=0$} & \multicolumn{4}{|c|}{$[g, h]=0, g \neq 0$} \\
\hline & & & \multirow[t]{2}{*}{$\gamma \neq 0$} & \multirow[t]{2}{*}{$\gamma=0$} & \multicolumn{2}{|l|}{$\gamma \neq 0$} & \multicolumn{2}{|l|}{$\gamma=0$} \\
\hline & & & & & $(\ell, g) \neq 0$ & $(\ell, g)=0$ & $(\ell, g) \neq 0$ & $(\ell, g)=0$ \\
\hline$\Gamma$ & 8 & 7 & 7 & 6 & 6 & 4 & 5 & 3 \\
\hline C & 4 & 4 & 3 & 3 & 3 & 2 & 3 & 2 \\
\hline E & 1 & 1 & 1 & 1 & 1 & 0 & 1 & 0 \\
\hline
\end{tabular}

Note that $E$ is in most cases elliptic. The genus of $C$ falls down from 4 to 3 in two cases characterized by the existence of an additional $S O(2)$-symmetry of the system.

Let us now turn to the original Kowalewski top where $h=0, \gamma=0$, and discuss the related curves in more detail. With no loss of generality we shall assume in the sequel that $|g|^{2}=1$.

The covering $\Gamma \rightarrow C$ is unramified and so is determined by a cycle $Z(\bmod 2)$ on $C$ : a loop $\gamma$ on $C$ lifts to a closed loop on $\Gamma$ if and only if $\langle\gamma, Z\rangle=0(\bmod 2)$, where $\langle\gamma, Z\rangle$ is the intersection number of $\gamma$ and $Z$. To put it another way, the function $\lambda=\sqrt{z}$ acquires a factor $(-1)^{\langle\gamma, z\rangle}$ upon a circuit of $\gamma$.

The curve $C$ can be thought of as the Riemann surface of the algebraic function $\mu=\sqrt{y}$ on $E$. The covering $C \rightarrow E$ defined by the involution $\pi:(\mu, z) \mapsto(-\mu, z)$ has 4 branch points. One can always choose a canonical basis $\left\{a_{i}, b_{i}\right\}, i=1,2,3$, in $H_{1}(C ; \mathbb{Z})$ so that

$$
\pi a_{1}=-a_{3}, \quad \pi b_{1}=-b_{3}, \quad \pi a_{2}=-a_{2}, \quad \pi b_{2}=-b_{2} .
$$

Lemma 5.1. The basis $\left\{a_{i}, b_{i}\right\}$ can be chosen in such a way that $a_{2}=\mathrm{Z}(\bmod 2)$.

For the proof, and also for later use, we must have a closer look at the covering $C \rightarrow E$. The elliptic curve $E$ is a two-sheeted cover of the $z$-plane. There are two points $\infty_{ \pm}$at the "infinity" where $z$ has simple poles, and one point 0 where $z$ has a double zero. The function $y$ has a simple pole at $\infty_{+}$, a simple zero at $\infty_{-}$, a double pole at 0 , and hence two other simple zeros at some points $p_{1}, p_{2}$. The branch points of the function $\mu=\sqrt{y}$ on $E$ are therefore $\infty_{+}, \infty_{-}, p_{1}, p_{2}$. Thus $C$ is obtained by glueing together two copies of $E$ along suitable cuts $\left[\infty_{+}, \infty_{-}\right]$and $\left[p_{1}, p_{2}\right]$.

We choose the cut $\left[\infty_{+}, \infty_{-}\right]$in such a way that the function $\lambda=\sqrt{z}$ becomes unramified on $E \backslash\left[\infty_{+}, \infty_{-}\right]$(notice that $\infty_{ \pm}$are the only branch points of $\sqrt{z}$ ).

The curve $\Gamma$ may be thought of as the Riemann surface of the function $\lambda=\sqrt{z}$ on $C$. Hence to determine the ramification cycle $\mathbf{Z}$ of the covering $\Gamma \rightarrow C$ we must

\footnotetext{
${ }^{1}$ Note that the curves in this table are nonsingular compactifications of the affine curves given by Eqs. (5.1), (5.3), and (5.4). For special values of the parameters the geometric genera of these curves drop down (while the arithmetic genera, of course, remain invariant)
} 


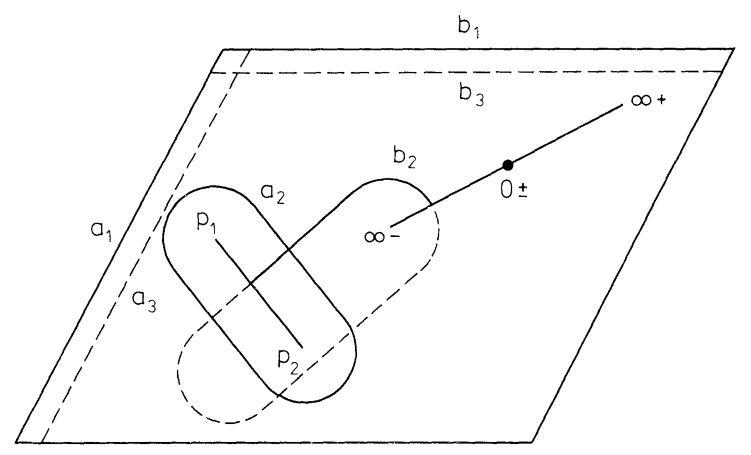

Fig. 1. Shows a plane model of the elliptic curve $E$. The cut $\left[\infty_{+}, \infty_{-}\right]$can always be placed inside the parallelogram; the location of the other cut $\left[p_{1}, p_{2}\right]$ depends on the topology of the covering $I \rightarrow E$. The cycles $a_{i}, b_{i}$ are depicted relative to the representation of $C$ as a two-sheeted cover of $E$ : continuous lines show parts of the cycles on the upper sheet while dotted lines show their parts on the lower sheet

know the behaviour of $\lambda$ over the cycles $a_{i}, b_{i}$. Since $\sqrt{z}$ is unramified on $E \backslash\left[\infty_{+}, \infty_{-}\right]$, it is obvious from Fig. 1 that $\lambda$ changes sign when we make a circuit of $b_{2}$, and does not ramify over all other basis cycles. Therefore $\mathbf{Z}=a_{2}(\bmod 2)$, as was to be shown.

We may now identify $\Gamma$ with two copies of $C$ glued together along $\mathrm{Z}: \Gamma=C^{(1)}$ $\cup_{Z} C^{(2)}$. It is natural to choose the contour $Z$ in such a way that $\pi Z=-Z$ (the minus sign denotes reversed orientation). Note that there are well-defined branches of $\lambda=\sqrt{z}$ on $C^{(i)} \backslash Z$. The involution $\tau_{1}$ acts on $\Gamma$ by permuting the sheets $C^{(i)}$.

The final thing we need is the behaviour of $\mu$ near the points of $\Gamma$ where $\lambda=\infty$ or $\lambda=0$. These are the points $\infty_{ \pm}^{(i)}$ and $0_{ \pm}^{(i)}$ on the sheets $C^{(i)}$. The curve $\Gamma$ is unramified at $\lambda=\infty$ and $\lambda=0$ over the $\lambda$-plane; $\lambda^{-1}$ is a local parameter at $\infty_{+}^{(i)}$ and $\lambda$ is a local parameter at $0_{ \pm}^{(i)}$. If we arrange the points $\infty_{ \pm}^{(i)}$ into a 4-tuple $\left(\infty_{-}^{(1)}, \infty_{-}^{(2)}\right.$, $\left.\infty_{+}^{(1)}, \infty_{+}^{(2)}\right)$, the 4 branches of $\mu$ near $\lambda=\infty$ can be combined into a row-vector

$$
\mu(\lambda) \sim(0,0,2 \lambda,-2 \lambda),+o(1) .
$$

In a similar way, with respect to the ordering $\left(0_{-}^{(1)}, 0_{+}^{(2)}, 0_{+}^{(1)}, 0_{-}^{(2)}\right)$ (this particular ordering is convenient for the calculations in Sect. 7.4) we have

$$
\mu(\lambda) \sim-\varepsilon \lambda^{-1}(1,-1,1,-1)
$$

near $\lambda=0$, with $\varepsilon= \pm 1$ depending on the location of $Z$ (recall that $|g|^{2}=1$ ). It always possible to choose $Z$ in such a way that $\varepsilon=1$.

\section{Analyticity Properties of the Baker-Akhiezer Function}

The principal tool of finite-gap integration techniques is the Baker-Akhiezer function defined as a solution of the linear system

$$
L(\lambda(p)) \psi(p)=\mu(p) \psi(p), \quad \frac{\partial}{\partial t} \psi(p)=-M(\lambda(p)) \psi(p)
$$


which has certain analyticity properties as a vector-valued function on $\Gamma$. We can also require $\psi$ to be symmetric with respect to the involution (4.8):

$$
\psi\left(\tau_{1} p\right)=\eta \psi(p) .
$$

This enables us to regard $\psi$ as a double-valued function on the curve $C=\Gamma / \tau_{1}$, which makes all calculations much simpler.

We continue to deal with the classical Kowalewski case: $h=0, \gamma=0$. It is convenient to express the Lax pair (1.7)-(1.8) in a basis in which $\eta$ is diagonal, i.e., to conjugate it by the matrix $\left(\begin{array}{cc}\varrho & 0 \\ 0 & -\varrho\end{array}\right), \varrho=\left(\begin{array}{cc}1 & -i \\ -i & 1\end{array}\right)$. We get the new Lax pair

$$
\begin{aligned}
L(\lambda)= & i\left(\begin{array}{cccc}
0 & g_{1}-i g_{2} & 0 & -g_{3} \\
-g_{1}-i g_{2} & 0 & g_{3} & 0 \\
0 & -g_{3} & 0 & -g_{1}-i g_{2} \\
g_{3} & 0 & g_{1}-i g_{2} & 0
\end{array}\right) \lambda^{-1} \\
& +i\left(\begin{array}{cccc}
0 & 0 & \ell_{1}-i \ell_{2} & 0 \\
0 & 0 & 0 & -\ell_{1}-i \ell_{2} \\
\ell_{1}+i \ell_{2} & 0 & -2 \ell_{3} & -2 \lambda \\
0 & -\ell_{1}+i \ell_{2} & 2 \lambda & 2 \ell_{3}
\end{array}\right), \\
M(\lambda)= & -\frac{i}{2}\left(\begin{array}{cccc}
2 \ell_{3} & 0 & \ell_{1}-i \ell_{2} & 0 \\
0 & -2 \ell_{3} & 0 & -\ell_{1}-i \ell_{2} \\
\ell_{1}+i \ell_{2} & 0 & -2 \ell_{3} & -2 \lambda \\
0 & -\ell_{1}+i \ell_{2} & 2 \lambda & 2 \ell_{3}
\end{array}\right) .
\end{aligned}
$$

The matrix $\eta$ in (6.2) becomes

$$
\eta=\operatorname{diag}(1,-1,1,-1)=\left(\begin{array}{cc}
\sigma_{3} & 0 \\
0 & \sigma_{3}
\end{array}\right) .
$$

We recall the definition of the Pauli matrices $\sigma_{i}$ :

$$
\sigma_{1}=\left(\begin{array}{ll}
0 & 1 \\
1 & 0
\end{array}\right), \quad \sigma_{2}=\left(\begin{array}{cc}
0 & -i \\
i & 0
\end{array}\right), \quad \sigma_{3}=\left(\begin{array}{cc}
1 & 0 \\
0 & -1
\end{array}\right)
$$

The new matrix $L(\lambda)$ satisfies the following symmetry relations:

$$
\begin{gathered}
L(-\lambda)=\eta L(\lambda) \eta \\
L(\lambda)^{t}=-\left(\begin{array}{cc}
\sigma_{2} & 0 \\
0 & \sigma_{2}
\end{array}\right) L(\lambda)\left(\begin{array}{cc}
\sigma_{2} & 0 \\
0 & \sigma_{2}
\end{array}\right) .
\end{gathered}
$$

Let us now state the analyticity properties of $\psi$ which specialize the general properties of the Baker-Akhiezer function outlined in Sect. 4.

1. $\psi$ is meromorphic on $\Gamma$ except at $\lambda=\infty$ and $\psi \exp (-t \mu / 2)$ is meromorphic on $\Gamma$ except at $\lambda=0$ (recall that in our case $d H(L)=1 / 2 L$, so that $v=\mu / 2$ ).

2. The divisor of poles of $\psi$, denoted by $\hat{\mathscr{D}}$, has degree 8 and is time-independent (points where $\lambda=\infty$ enter in $\hat{\mathscr{D}}$ if $\psi \exp (-t \mu / 2)$ has poles there).

3. $\psi$ satisfies the symmetry condition (6.2). 
The divisor $\hat{\mathscr{D}}$ is not however completely determined by these conditions. If $f$ is a meromorphic function on $\Gamma$ such that $f\left(\tau_{1} p\right)=f(p)$ and $(f) \leqq \hat{\mathscr{D}}$, then $\psi$ can be replaced by $f \psi$. Using this freedom we can fix two points of $\mathscr{D}$ to be $\infty_{+}^{1}$ and $\infty_{+}^{2}$. Then $\hat{\mathscr{D}}$ is the pull-back to $\Gamma$ of a divisor $\mathscr{D} \cup \infty_{+}$on $C$, and $\operatorname{deg} \mathscr{D}=3$.

The behaviour of $\psi$ near $\lambda=\infty$ can be reformulated in a more convenient matrix form. Let $\Psi(\lambda)$ be the $4 \times 4$ matrix whose $j^{\text {th }}$ column is the value of $\psi$ on the $j^{\text {th }}$ sheet of $\Gamma \rightarrow\{\lambda\}$ near $\lambda=\infty$ [the ordering of sheets corresponds to the ordering of points over $\lambda=\infty$ described in Sect. 5, cf. (5.6)]. We can then write $\Psi(\lambda)$ as

$$
\Psi(\lambda, t)=\left(\Phi(t)+S(t) \lambda^{-1}+\ldots\right) \operatorname{diag}\left(1,1, \lambda e^{\lambda t},-i \lambda e^{-\lambda t}\right)
$$

where the matrices $\Phi(t), S(t)$ do not depend on $\lambda$ [the factor $-i$ in the last entry of (6.9) is taken for notational convenience]. Denoting

$$
\begin{aligned}
L(\lambda) & =L_{-1} \lambda^{-1}+L_{0}+L_{1} \lambda, \\
M(\lambda) & =M_{0}+M_{1} \lambda,
\end{aligned}
$$

we have from (6.1), (6.9), and (5.6),

$$
\begin{gathered}
L_{1}=2 \Phi\left(\begin{array}{cc}
0 & 0 \\
0 & \sigma_{3}
\end{array}\right) \Phi^{-1}, \\
L_{0}=\left[S \Phi^{-1}, L_{1}\right], \\
M_{1}=-\Phi\left(\begin{array}{cc}
0 & 0 \\
0 & \sigma_{3}
\end{array}\right) \Phi^{-1}, \\
M_{0}=-\partial_{t} \Phi \Phi^{-1}+\left[S \Phi^{-1}, M_{1}\right] .
\end{gathered}
$$

The symmetry condition (6.2) takes the form (notice that $\tau_{1}$ permutes the sheets):

$$
\Psi(-\lambda)=\left(\begin{array}{cc}
\sigma_{3} & 0 \\
0 & \sigma_{3}
\end{array}\right) \Psi(\lambda)\left(\begin{array}{cc}
\sigma_{1} & 0 \\
0 & \sigma_{1}
\end{array}\right)
$$

which gives the symmetry relations for $\Phi$ and $S$ :

$$
\left(\begin{array}{cc}
\sigma_{3} & 0 \\
0 & \sigma_{3}
\end{array}\right) \Phi\left(\begin{array}{cc}
\sigma_{1} & 0 \\
0 & \sigma_{2}
\end{array}\right)=\Phi, \quad\left(\begin{array}{cc}
\sigma_{3} & 0 \\
0 & \sigma_{3}
\end{array}\right) S\left(\begin{array}{cc}
\sigma_{1} & 0 \\
0 & \sigma_{2}
\end{array}\right)=-S
$$

Combined with (6.11) this implies that $\Phi(t)$ has the form

$$
\Phi(t)=c \cdot \operatorname{diag}\left(q_{1}(t), q_{2}(t), 1,1\right) \cdot\left(\begin{array}{cccc}
1 & 1 & 0 & 0 \\
1 & -1 & 0 & 0 \\
0 & 0 & 1 & -i \\
0 & 0 & -i & 1
\end{array}\right)
$$

We can set $c=1$. Relation $\left(6.12^{\prime}\right)$ yields differential equations for $q_{i}(t)$ :

$$
\begin{aligned}
& d q_{1} / d t=i \ell_{3} q_{1}, \\
& d q_{2} / d t=-i \ell_{3} q_{2},
\end{aligned}
$$


so that

$$
\begin{array}{r}
q_{1}(t)=\alpha \exp \left(i \int^{t} \ell_{3} d t^{\prime}\right), \\
q_{2}(t)=\beta \exp \left(-i \int^{t} \ell_{3} d t^{\prime}\right) .
\end{array}
$$

In a similar way, arranging the 4 eigenvectors $\psi\left(0_{ \pm}^{(i)}\right)$ into a $4 \times 4$ matrix $\Psi(0)$ according to the ordering of the points $0_{ \pm}^{(i)}$ described in Sect. 5, we have

$$
\left(\begin{array}{cc}
\sigma_{3} & 0 \\
0 & \sigma_{3}
\end{array}\right) \Psi(0)\left(\begin{array}{cc}
0 & \sigma_{1} \\
\sigma_{1} & 0
\end{array}\right)=\Psi(0)
$$

(cf. (6.13)) and using (5.7), (6.1) we find

$$
L_{-1}=-\varepsilon \Psi(0)\left(\begin{array}{cc}
\sigma_{3} & 0 \\
0 & \sigma_{3}
\end{array}\right) \Psi^{-1}(0)
$$

[recall that $\varepsilon$ is defined in (5.7)].

The strategy of our further computations will be as follows. Using the symmetry property (6.2) of $\psi$ we reformulate the problem entirely in terms of the curve $C$ : the components $\psi_{1}$ and $\psi_{3}$ are single-valued functions on $C$, whereas $\psi_{2}$ and $\psi_{4}$ are double-valued functions which acquire a factor $(-1)^{\langle\gamma, Z\rangle}$ upon a circuit of a loop $\gamma$ on $C$. The properties of $\psi$ stated above allow us to write explicit formulae for $\psi_{3}, \psi_{4}$ which in turn serve to compute the coefficients $S_{i j}$ of $S$ for $i, j=3,4$. From $\left(6.11^{\prime}\right)$ we have the relation

$$
\ell_{3}=-i S_{33}-S_{43}
$$

which by (6.17) yields expressions for $q_{1}(t), q_{2}(t)$. Remarkably, the integral in (6.17) can be evaluated in closed form. After that we can write down the remaining components $\psi_{1}, \psi_{2}$. To determine the constant factors that occur in these formulae we must use the second symmetry relation (6.8). Combining it with $\left(6.11^{\prime}\right)$ we come down to the Prym condition for the divisor $\mathscr{D}$ and determine the Baker-Akhiezer function completely. Finally, to derive the evolution of $g(t)$ we use (6.19).

\section{Explicit Formulae for the Solutions}

\subsection{Theta Functions and Abelian Differentials}

We now begin to implement the programme outlined above. First of all we have to introduce some standard notions.

Recall that Riemann's theta function with characteristics is defined by

$$
\theta\left[\begin{array}{l}
\alpha \\
\beta
\end{array}\right](z \mid B)=\sum_{m \in \mathbb{Z}^{g}} \exp \left\{\frac{1}{2}\langle B(m+\alpha), m+\alpha\rangle+\langle z+2 \pi i \beta, m+\alpha\rangle\right\}
$$

for a symmetric $g \times g$-matrix $B$ with $\operatorname{Re} B<0$.

Let $d u_{k}, k=1,2,3$, be the normalized abelian differentials on $C$ :

$$
\int_{a_{j}} d u_{k}=2 \pi i \delta_{j k}
$$


and let $B$ be the associated period matrix:

$$
B_{j k}=\int_{b_{j}} d u_{k} .
$$

We shall write $\theta(z)$ for $\theta\left[\begin{array}{l}0 \\ 0\end{array}\right](z \mid B)$.

Let $d \Omega$ be an abelian differential of the second kind on $C$ with a double pole at $\infty_{+}$such that $d \Omega-1 / 2 d \mu$ is regular at $\infty_{+}$and

$$
\int_{a_{t}} d \Omega=0
$$

We put

$$
\Omega(p)=\int^{p} d \Omega
$$

and fix the constant of integration in (7.5) by the condition

$$
\Omega(p)=\lambda+O\left(\lambda^{-1}\right) \text { as } p \rightarrow \infty_{+}
$$

(recall that there is a well-defined branch of $\lambda$ on $C \backslash Z$; its sign is specified by requiring that $\mu \sim 2 \lambda$ at $\left.\infty_{+}\right)$. We remind that the involution $\pi$ on $C$ acts by $\pi(z, \mu)$ $=(z,-\mu)$.

Lemma 7.1. (i)

$$
\pi^{*} d \Omega=-d \Omega
$$

(ii) Let

$$
V_{j}=\int_{b_{j}} d \Omega
$$

Then $V=\left(V_{1}, V_{2}, V_{3}\right)$ coincides with the velocity vector for the Kowalewski flow on $\mathrm{Jac} C$.

Proof. (i) The differential $\pi^{*} d \Omega$ has the same principal part as $-d \Omega$ at $p=\infty_{+}$. Since $\pi_{*} a_{i}= \pm a_{i}, \pi^{*} d \Omega$ also satisfies the normalization conditions (7.4) and hence $\pi^{*} d \Omega=-d \Omega$.

(ii) By the reciprocity law for abelian differentials [19] we have

$$
\int_{b_{j}} d \Omega=\operatorname{Res}_{\infty+} \Omega(p) d u_{j}(p)=\operatorname{Res}_{\infty+\frac{1}{2}} \mu d u_{\jmath},
$$

so that (ii) follows from (4.10).

Let $d \omega$ be an abelian differential of the third kind which has simple poles at $\infty_{+}$ and $\infty_{-}$with residues 1 and -1 , respectively. We choose a path $\ell$ from $\infty_{+}$to $\infty_{-}$ and normalize $d \omega$ by the condition

$$
\int_{a_{J}} d \omega=0
$$

where the cycles $a_{j}$ are supposed not to intersect $\ell$. It is easily checked that $d \omega$ is the pullback to $C$ of a differential on $E$ given by

$$
d \omega=\frac{\left(1+q z^{-1}\right) d z}{y-d_{1}(z)}
$$


with some constant $q$, so that $\pi^{*} d \omega=d \omega$. We put

$$
\omega(p)=\int^{p} d \omega
$$

and fix the constant of integration in (7.12) by the condition

$$
e^{\omega(p)}=\lambda+O(1) \text { as } p \rightarrow \infty_{+} .
$$

Let

$$
u(p)=\left(u_{1}(p), u_{2}(p), u_{3}(p)\right), \quad u_{j}(p)=\int_{\infty+\infty}^{p} d u_{j}
$$

be the Abel transform with origin $\infty_{+}$.

We shall need the values of the multi-valued functions $\Omega(p), e^{\omega(p)}$, and $u_{j}(p)$ at the points $\infty_{ \pm}$and $0_{ \pm}$. For that purpose we shall specify the choice of the path $\ell$ joining $\infty_{+}$and $\infty_{-}$by requiring that

a) $\ell$ passes through $0_{+}$;

b) the cycle $\ell-\pi \ell$ is homologous to $a_{2}$;

c) $\ell$ does not intersect the ramification contour $Z$.

Since the periods of $d \Omega, d \omega, d u_{1}$, and $d u_{3}$ over $a_{2}$ are all zero, the multi-valued analytic functions $\Omega(p), e^{\omega(p)}, u_{1}(p)$ and $u_{3}(p)$ have single-valued branches in a neighbourhood of the contour $\ell \cup \pi \ell$, determined by the conditions (7.6), (7.13), and $u_{J}\left(\infty_{+}\right)=0$. The function $u_{2}(p)$ has period $2 \pi i$ along $\ell \cup \pi \ell$.

\section{Lemma 7.2. Let}

$$
r=\int_{b_{1}} d \omega=-\int_{b_{3}} d \omega
$$

Then

$$
u\left(\infty_{-}\right)=\int_{\ell} d u=(r, \pi i,-r), \quad \int_{b_{2}} d \omega=\pi i .
$$

Proof. Since $\ell-\pi \ell=a_{2}$, and $\pi^{*} d u_{2}=-d u_{2}$, we have

$$
\int_{t} d u_{2}=\int_{t-a_{2}} \pi^{*} d u_{2}=-\int_{t} d u_{2}+2 \pi i,
$$

hence $\int_{\ell} d u_{2}=\pi i$. Then the reciprocity law for abelian differentials

$$
\int_{b_{j}} d \omega=\int_{\ell} d u_{j}
$$

gives (7.16).

In particular, $e^{\omega(p)}$ changes sign when analytically continued along $b_{2}$.

We shall also need the following lemma.

\section{Lemma 7.3.}

(i) $\Omega\left(\infty_{-}\right)=0$.

(ii) $e^{\omega\left(0_{+}\right)}=-e^{\omega\left(0_{-}\right)}$.

(iii) $e^{\omega(p)}=-\frac{a^{2}}{\lambda}+O(1)$ as $p \rightarrow \infty_{-}$; 
if the path $\ell$ joining $\infty_{+}$and $\infty_{-}$is such that its projection to $E$ is symmetric with respect to $0 \in E$, then $a^{2}=e^{2 \omega\left(0_{+}\right)}$. It will be convenient for us to put $a=e^{\omega\left(0_{+}\right)}$.

Proof. (i) In view of (7.6) and (7.7) we have $\Omega(\pi p)=-\Omega(p)$ in the vicinity of $\ell \cup \pi \ell$. Since $\pi \infty_{-}=\infty_{-},(7.17)$ follows.

(ii) We only have to observe that $e^{\omega(\pi p)}=-e^{\omega(p)}$ near $\ell \cup \pi \ell$.

(iii) We consider $d \omega$ as a differential on $E$ and let $\varrho$ be the symmetry of $E$ given by reflection at $0 \in E$ (this is the involution that permutes the roots $y_{1}, y_{2}$ of Eq. (5.4): see Fig. 1). Then $\varrho^{*} d \omega=-d \omega$, so that $\int_{0}^{\varrho p} d \omega=-\int_{0}^{p} d \omega$. Together with $\lambda(\varrho p)=-\lambda(p)$ and $\varrho \infty_{+}=\infty_{\text {_ }}$ this easily leads to (7.19).

\subsection{The Baker-Akhiezer Function}

Let $D$ be a vector in $\mathbb{C}^{3}$ such that the divisor of $\theta(u(p)+D)$ on $C$ coincides with the divisor $\mathscr{D}$ introduced above.

Proposition 7.4. The functions $\varphi_{i}(p, t)$ defined by

$$
\begin{aligned}
\varphi_{1} & =\frac{\theta(u(p)+V t+D) \theta[\varepsilon](D+R)}{\theta(u(p)+D) \theta[\varepsilon](V t+D+R)} e^{\Omega(p) t}, \\
\varphi_{2} & =\frac{\theta[\varepsilon](u(p)+V t+D) \theta[\varepsilon](D+R)}{\theta(u(p)+D) \theta(V t+D+R)} e^{\Omega(p) t}, \\
\varphi_{3} & =\frac{\theta[\varepsilon](u(p)+V t+D+R) \theta(D)}{\theta(u(p)+D) \theta[\varepsilon](V t+D+R)} e^{\omega(p)+\Omega(p) t}, \\
\varphi_{4} & =-i \frac{\theta(u(p)+V t+D+R) \theta(D)}{\theta(u(p)+D) \theta(V t+D+R)} e^{\omega(p)+\Omega(p) t},
\end{aligned}
$$

where

$$
R=(r, 0,-r), \quad \varepsilon=\left[\begin{array}{ccc}
(0 & 0 & 0 \\
(0 & 1 / 2 & 0
\end{array}\right],
$$

have the following analyticity properties:

(i) $\varphi_{1}, \varphi_{3}$ are single-valued on $C$ while $\varphi_{2}, \varphi_{4}$ are double-valued on $C$ and acquire a factor $(-1)^{\left\langle\gamma, a_{2}\right\rangle}$ when analytically continued along a closed path $\gamma$.

(ii) $\left(\varphi_{1}, \varphi_{2}, \varphi_{3}, \varphi_{4}\right)\left(\infty_{-}\right)=(1,1,0,0)$,

$$
\left(\varphi_{1}, \varphi_{2}, \varphi_{3}, \varphi_{4}\right)(p)=((0,0,1,-i) \lambda+O(1)) e^{\lambda t}, \quad p \rightarrow \infty_{+} .
$$

(iii) The divisor of poles of $\varphi_{i}$ in the affine part of $C$ coincides with $\mathscr{D}$.

The proof is straightforward and follows from the periodicity properties of $\theta(u)$ and the analyticity properties of $\Omega(p), \omega(p)$ displayed above.

There is an obvious relationship between the $\varphi_{i}$ and the components $\psi_{i}$ of the Baker-Akhiezer function:

$$
\begin{array}{ll}
\psi_{1}=q_{1} \varphi_{1}, & \psi_{3}=\varphi_{3}, \\
\psi_{2}=q_{2} \varphi_{2}, & \psi_{4}=\varphi_{4} .
\end{array}
$$


The expressions (7.20) for $\psi_{3}=\varphi_{3}$ and $\psi_{4}=\varphi_{4}$ enable us to calculate $\ell_{3}(t)$.

\section{Lemma 7.5.}

$$
\ell_{3}(t)=-i \frac{\partial}{\partial t} \log \frac{\theta[\varepsilon](V t+D+R)}{\theta(V t+D+R)} .
$$

Proof. From (6.20) we get

$$
\begin{gathered}
\ell_{3}(t)=\lim _{p \rightarrow \infty_{+}} \lambda\left(-i \psi_{3}(p, t)-\psi_{4}(p, t)\right) \\
=-i\left[\frac{\frac{\partial}{\partial k} \theta[\varepsilon](u(p)+V t+D+R)}{\theta[\varepsilon](V t+D+R)}-\frac{\frac{\partial}{\partial k} \theta(u(p)+V t+D+R)}{\theta(V t+D+R)}\right],
\end{gathered}
$$

where $k=\lambda^{-1}$ is a local parameter at $p=\infty_{+}$. Recall now the formula (4.10) for the velocity vector of the Kowalewski flow. Let $d u_{j}=f_{i}(k) d k$. Clearly, (4.10) implies that $V=f(0)$. On the other hand, the Abel transform near $p=\infty_{+}$is given by

$$
u(p)=\int_{\infty+\infty}^{p} d u=f(0) k+O\left(k^{2}\right) .
$$

Hence the dervative $\partial / \partial k$ in (7.23) at $k=0$ may be replaced by $\partial / \partial t$, which yields (7.22).

We can now substitute (7.22) into (6.17) to find the $q_{i}(t)$. The integrand in (6.17) turns out to be an exact derivative and so we get

$$
\begin{aligned}
& q_{1}(t)=\alpha \frac{\theta[\varepsilon](V t+D+R)}{\theta(V t+D+R)}, \\
& q_{2}(t)=\beta \frac{\theta(V t+D+R)}{\theta[\varepsilon](V t+D+R)},
\end{aligned}
$$

where the constants of integration $\alpha, \beta$ are still to be determined. This gives the following expressions for the Baker-Akhiezer function.

Proposition 7.6. The Baker-Akhiezer function $\psi(p, t)$ is given by

$$
\begin{gathered}
\psi_{1}=\alpha \frac{\theta(u(p)+V t+D) \theta[\varepsilon](D+R)}{\theta(u(p)+D) \theta(V t+D+R)} e^{\Omega(p) t}, \\
\psi_{2}=\beta \frac{\theta[\varepsilon](u(p)+V t+D) \theta[\varepsilon](D+R)}{\theta(u(p)+D) \theta[\varepsilon](V t+D+R)} e^{\Omega(p) t}, \\
\psi_{3}=\varphi_{3}, \\
\psi_{4}=\varphi_{4} .
\end{gathered}
$$

\subsection{The Prym Condition}

It is now time to take into account the second symmetry condition (6.8), which is best done in the resultant formulae for the solutions. Substituting (7.25), (7.20) into 
$\left(6.11^{\prime}\right)$ gives the coefficients of $L_{0}$ :

$$
\begin{aligned}
& \left(L_{0}\right)_{13}=-2 S_{13}=-2 \alpha \frac{\theta(V t+D) \theta[\varepsilon](D+R)}{\theta(V t+D+R) \theta(D)}, \\
& \left(L_{0}\right)_{42}=-2 i S_{31} q_{2}^{-1}=\frac{2 i}{\beta} a^{2} \frac{\theta(V t+D+2 R) \theta(D)}{\theta(V t+D+R) \theta[\varepsilon](D+R)}, \\
& \left(L_{0}\right)_{24}=-2 i S_{23}=-2 i \beta \frac{\theta[\varepsilon](V t+D) \theta[\varepsilon](D+R)}{\theta[\varepsilon](V t+D+R) \theta(D)}, \\
& \left(L_{0}\right)_{31}=2 i S_{41} q_{1}^{-1}=-\frac{2 a^{2}}{\alpha} \frac{\theta[\varepsilon](V t+D+2 R) \theta(D)}{\theta[\varepsilon](V t+D+R) \theta[\varepsilon](D+R)} .
\end{aligned}
$$

Relation (6.8) implies $\left(L_{0}\right)_{13}=-\left(L_{0}\right)_{42},\left(L_{0}\right)_{24}=-\left(L_{0}\right)_{31}$, which gives

$$
\alpha \beta=i a^{2} \frac{\theta(D)^{2} \theta(V t+D+2 R)}{\theta(D+R)^{2} \theta(V t+D)}=i a^{2} \frac{\theta(D)^{2} \theta[\varepsilon](V t+D+2 R)}{\theta(D+R)^{2} \theta[\varepsilon](V t+D)}
$$

[recall that $a^{2}$ is defined by (7.19)]. For this equality to hold identically, the theta functions depending on $t$ must cancel out:

$$
\theta(V t+D+2 R)=c \theta(V t+D)
$$

with some constant $c$. Since $\pi^{*} V=-V, \pi^{*} R=R$ and moreover,

$$
\theta(-u)=\theta(u), \quad \theta\left(\pi^{*} u\right)=\theta(u)
$$

this implies

$$
\pi^{*} D+D=-2 R \text { (modulo periods). }
$$

It is not hard to show that one can add a period to $D$ so as to make (7.28) an exact equality. This choice of $D$ enters in all the subsequent formulae. Substituting

$$
D=P-R,
$$

we have

$$
\pi^{*} P=-P
$$

The relations (7.29), (7.30) imply that

$$
\begin{gathered}
\alpha=\Delta a \frac{\theta(P-R)}{\theta[\varepsilon](P)}, \quad \beta=\frac{i a}{\Delta} \frac{\theta(P-R)}{\theta[\varepsilon](P)}, \\
\ell_{1}-i \ell_{2}=2 i \Delta a \frac{\theta(V t+P-R)}{\theta(V t+P)}, \\
\ell_{1}+i \ell_{2}=\frac{2 i a}{\Delta} \frac{\theta[\varepsilon](V t+P-R)}{\theta[\varepsilon](V t+P)},
\end{gathered}
$$

where the constant $\Delta$ is still to be determined.

\subsection{The Poisson Vector}

It remains to calculate the Poisson vector $g(t)$ using (6.19); recall that $g_{1}^{2}+g_{2}^{2}$ $+g_{3}^{2}=1$. In view of (6.18), we can write $\Psi(0)$ as

$$
\Psi(0)=\mathscr{L}\left(\begin{array}{ll}
A & \sigma_{3} A \sigma_{1} \\
B & \sigma_{3} B \sigma_{1}
\end{array}\right) \mathscr{R}
$$


where

$$
\begin{aligned}
\mathscr{L}= & \operatorname{diag}\left(\frac{\Delta}{\theta(V t+P)},-\frac{i}{\Delta \theta[\varepsilon](V t+P)},-\frac{1}{\theta[\varepsilon](V t+P)}, \frac{i}{\theta(V t+P)}\right), \\
\mathscr{R}= & a \theta(P-R) \\
& \times \operatorname{diag}\left(\frac{e^{\Omega\left(0_{-}\right) t}}{\theta\left(u\left(0_{-}\right)+P-R\right)}, \frac{e^{\Omega\left(0_{+}\right) t}}{\theta\left(u\left(0_{+}\right)+P-R\right)},\right. \\
& \left.\frac{e^{\Omega\left(0_{+}\right) t}}{\theta\left(u\left(0_{+}\right)+P-R\right.}, \frac{e^{\Omega\left(0_{-}\right) t}}{\theta\left(u\left(0_{-}\right)+P-R\right)}\right) \\
\mathscr{A}= & \left(\begin{array}{cc}
\theta\left(u\left(0_{-}\right)+V t+P-R\right) & \theta\left(u\left(0_{+}\right)+V t+P-R\right) \\
\theta[\varepsilon]\left(u\left(0_{-}\right)+V t+P-R\right) & -\theta[\varepsilon]\left(u\left(0_{+}\right)+V t+P-R\right)
\end{array}\right), \\
\mathscr{B}= & \left(\begin{array}{cc}
\theta[\varepsilon]\left(u\left(0_{-}\right)+V t+P\right) & -\theta[\varepsilon]\left(u\left(0_{+}\right)+V t+P\right) \\
\theta\left(u\left(0_{-}\right)+V t+P\right) & \theta\left(u\left(0_{+}\right)+V t+P\right)
\end{array}\right) .
\end{aligned}
$$

To verify these formulae recall that $\psi_{j}\left(0_{ \pm}^{(1)}\right)=(-1)^{j} \psi_{j}\left(0_{ \pm}^{(2)}\right)$ and $e^{\omega\left(0_{ \pm}\right)}= \pm a$. Also, it can easily be shown that

$$
u\left(0_{ \pm}\right)=\frac{1}{2} R \pm C, \quad \pi^{*} C=-C .
$$

This and the relation $\theta(u)=\theta\left(-\pi^{*} u\right)$ imply

$$
\begin{gathered}
\theta\left(u\left(0_{ \pm}\right)+V t+P-R\right)=\theta\left(u\left(0_{ \pm}\right)+V t+P\right), \\
\theta[\varepsilon]\left(u\left(0_{ \pm}\right)+V t+P-R\right)=\theta[\varepsilon]\left(u\left(0_{ \pm}\right)+V t+P\right),
\end{gathered}
$$

so that

$$
\mathscr{B}=\sigma_{1} \mathscr{A}
$$

Therefore (6.19) can be written as

$$
L_{-1}=-\mathscr{L} W\left(\begin{array}{cc}
\sigma_{3} & 0 \\
0 & \sigma_{3}
\end{array}\right) W^{-1} \mathscr{L}^{-1}
$$

with

$$
W=\left(\begin{array}{cc}
\mathscr{A} & \sigma_{3} \mathscr{A} \sigma_{1} \\
\sigma_{1} \mathscr{A} & \sigma_{3} \sigma_{1} \mathscr{A} \sigma_{1}
\end{array}\right), \quad W^{-1}=\frac{1}{2}\left(\begin{array}{cc}
\mathscr{A}^{-1} & \mathscr{A}^{-1} \sigma_{1} \\
\sigma_{1} \mathscr{A}^{-1} \sigma_{3} & -\sigma_{1} \mathscr{A}^{-1} \sigma_{1} \sigma_{3}
\end{array}\right)
$$

[we have assumed that $\varepsilon=1$ in (6.19), which can always be achieved by a suitable choice of the contour Z, cf. (5.7)]. After simple calculations we find

$$
L_{-1}=-\mathscr{L}\left(\begin{array}{cc}
S_{1} \sigma_{1}+S_{2} \sigma_{2} & S_{3} \sigma_{3} \sigma_{1} \\
S_{1} \sigma_{1} \sigma_{3} & S_{1} \sigma_{1}-S_{2} \sigma_{2}
\end{array}\right) \mathscr{L}^{-1}
$$

where the $S_{j}$ are defined by

$$
\sum S_{j} \sigma_{j}=\mathscr{A} \sigma_{3} \mathscr{A}^{-1}
$$


By equating the matrix coefficients $\left(L_{-1}\right)_{32}=\left(L_{-1}\right)_{14}$ we finally get $\Delta^{2}=1$ and

$$
\begin{aligned}
g_{1}-i g_{2} & =\frac{\theta[\varepsilon](V t+P)}{\theta(V t+P)}\left(S_{1}-i S_{2}\right), \\
g_{1}+i g_{2} & =\frac{\theta(V t+P)}{\theta[\varepsilon](V t+P)}\left(S_{1}+i S_{2}\right), \\
g_{3} & =-\Delta S_{3} .
\end{aligned}
$$

We can now sum up our calculations.

Theorem 7.7. The general solution of the equations of motion for the Kowalewski top is given by

$$
\begin{aligned}
\ell_{1}-i \ell_{2} & =2 i a \frac{\theta(V t+P-R)}{\theta(V t+P)}, \\
\ell_{1}+i \ell_{2} & =2 i a \frac{\theta[\varepsilon](V t+P-R)}{\theta[\varepsilon](V t+P)}, \\
\ell_{3} & =-i \frac{\partial}{\partial t} \log \frac{\theta[\varepsilon](V t+P)}{\theta(V t+P)}, \\
g_{1}-i g_{2} & =2 \frac{\theta[\varepsilon](V t+P)}{\theta(V t+P)} \cdot \frac{A B}{A D+B C}, \\
g_{1}+i g_{2} & =2 \frac{\theta(V t+P)}{\theta[\varepsilon](V t+P)} \cdot \frac{C D}{A D+B C}, \\
g_{3} & =-\frac{A D-B C}{A D+B C},
\end{aligned}
$$

where

$$
\begin{aligned}
\varepsilon=\left[\begin{array}{ccc}
(0 & 0 & 0) \\
(0 & 1 / 2 & 0
\end{array}\right), & a=e^{\omega\left(0_{+}\right)} \text {and } \\
A=\theta\left(u\left(0_{-}\right)+V t+P\right), & B=\theta\left(u\left(0_{+}\right)+V t+P\right), \\
C=\theta[\varepsilon]\left(u\left(0_{-}\right)+V t+P\right), & D=\theta[\varepsilon]\left(u\left(0_{+}\right)+V t+P\right) .
\end{aligned}
$$

Remark. The polar singularities of the Poisson vector $g(t)$ are in fact the same as those of the angular momentum $\ell(t)$ (see Sect. 7.6).

\subsection{The Geometry of the Liouville Tori}

The remaining indeterminacy in the expressions (7.42) for the dynamical variables (the change of sign $\Delta \mapsto-\Delta$ and the permutation $0_{+} \mapsto 0_{-}$) reflects the freedom in reconstructing the Lax matrix (6.3) from the algebraic data. It is easily verified that this freedom amounts to conjugation, $L \mapsto A L A^{-1}$, by a matrix $A$ of the form

$$
A=\operatorname{diag}(1,1,-1,-1) \text {. }
$$


This is equivalent to a renormalization of the Baker-Akhiezer function $\psi \mapsto A \psi$ and induces a symmetry of the Kowalewski top:

$$
\begin{aligned}
\ell & \mapsto B \ell, \quad g \mapsto-B g, \\
B & =\operatorname{diag}(-1,-1,1) .
\end{aligned}
$$

Clearly, (7.45) leaves the Hamiltonian invariant but changes the sign of $F_{1}=(\ell, g)$. Recall that only the square $I_{1}=F_{1}^{2}$ is a spectral invariant.

We may summarize the situation as follows.

Theorem 7.8. If $I_{1} \neq 0$, the common level surface of the spectral invariants $H, I_{1}, I_{2}$ consists of two components (Liouville tori) each of which is an affine part of an abelian variety isomorphic to Prym $C / E$. These components are permuted by the transformation (7.45).

If $I_{1}=0$, the level surface is irreducible. In this case the curve $E$ is rational, $C$ has genus 2, and Prym $C / E$ coincides with Jac $C$. The mapping of the Liouville torus to Jac $C$ becomes an unramified two-sheeted covering (for more details see Sect. 7.9).

\subsection{Complete Description of the Motion of the Kowalewski Top}

The solution (7.42) of the Euler-Poisson equations does not contain enough information to determine the rotation of the complete top's frame $k(t) \in S O(3)$. To find it we still have to solve the linear differential equation

$$
k^{-1} \frac{d k}{d t}=\omega(t)
$$

with initial condition $k(0)=k_{0}$ supplemented by the known evolution of the Poisson vector (assuming $e_{3}$ to be the gravity vector in the rest frame):

$$
k^{-1}(t) e_{3}=g(t)
$$

It turns out, however, that the Baker-Akhiezer function for the Lax pair (6.3)-(6.4) contains more information than the Euler-Poisson equations themselves and allows us to find $k(t)$ without solving (7.46). The point is that $\psi(\lambda)$ satisfies (at $\lambda=0)$ the equation

$$
\frac{d \psi}{d t}=-M_{0} \psi
$$

where $M_{0}$ essentially coincides with the angular velocity $\omega$ (see (6.4)). To make this more precise, observe that $M_{0}$ decomposes into two $2 \times 2$-blocks in the subspaces spanned by the standard basis vectors in $\mathbb{C}^{4}$ with odd (even) indices. (This corresponds to the decomposition of $\mathbb{C}^{4}$ with respect to the action of $s o(3)$ C so( $(3,2)$.) The first of these blocks is given by

$$
\hat{\omega}=\frac{1}{2 i} \sum_{j} \omega_{j} \sigma_{j} .
$$

Here and below we use the standard spinor notation for traceless $2 \times 2$-matrices

$$
\hat{X}=\frac{1}{2 i} \sum_{j} X_{j} \sigma_{j}, \quad X \in \mathbb{C}^{3} .
$$


It follows from (7.48) that the matrix

$$
\varphi=\left(\begin{array}{ll}
\psi_{1}\left(0_{-}\right) & \psi_{1}\left(0_{+}\right) \\
\psi_{3}\left(0_{-}\right) & \psi_{3}\left(0_{+}\right)
\end{array}\right)
$$

satisfies

$$
\frac{d \varphi}{d t}=-\hat{\omega} \varphi
$$

From (7.34) we find

$$
\begin{aligned}
\varphi= & a \theta(P-R)\left(\begin{array}{cc}
\frac{1}{\theta(V t+P)} & 0 \\
0 & -\frac{1}{\theta[\varepsilon](V t+P)}
\end{array}\right) \\
& \times \mathscr{A}\left(\begin{array}{cc}
\frac{e^{b t}}{\theta\left(u\left(0_{-}\right)+P-R\right)} & 0 \\
0 & \bar{\theta}\left(u\left(0_{+}\right)+P-R\right)
\end{array}\right),
\end{aligned}
$$

where $\pm b=\Omega\left(0_{+}\right)$and $\mathscr{A}$ is given by

$$
\mathscr{A}=\left(\begin{array}{cc}
\theta\left(u\left(0_{-}\right)+V t+P-R\right) & \theta\left(u\left(0_{+}\right)+V t+P-R\right) \\
\theta[\varepsilon]\left(u\left(0_{-}\right)+V t+P\right) & -\theta[\varepsilon]\left(u\left(0_{+}\right)+V t+P\right)
\end{array}\right) .
$$

Notice that in view of (7.36) $\mathscr{A}$ can also be written as in (7.34). It is easily checked that the time evolution of the Poisson vector $g(t)$ is given by

$$
\hat{g}(t)=\frac{1}{2 i} \varphi(t) \sigma_{3} \varphi(t)^{-1}
$$

An arbitrary solution of (7.52) satisfying (7.55) may differ from $\varphi(t)$ by a constant gauge factor

$$
\varphi^{\prime}(t)=\varphi(t) C, \quad\left[C, \sigma_{3}\right]=0 .
$$

Recall that the rest frame of the top is chosen in such a way that the gravity vector is directed along $e_{3}$. The evolution of two other vectors $e_{1}, e_{2}$ is given in the moving frame by

$$
\hat{e}_{j}(t)=\frac{1}{2 i} \varphi(t) C \sigma_{j} C^{-1} \varphi(t)^{-1}
$$

The remaining freedom (7.56) in $\varphi(t)$ corresponds to the (so far unspecified) initial conditions which indicate the relative positions of the two frames at $t=0$. By inverting we also obtain the evolution of the top in the rest frame. For example, the motion of the symmetry axis $s(t)$ of the top in the rest frame is given by

$$
\hat{s}(t)=\frac{1}{2 i}\left(\begin{array}{cc}
e^{-b t-b_{0}} & 0 \\
0 & e^{b t+b_{0}}
\end{array}\right) \mathscr{A}^{-1} \sigma_{3} \mathscr{A}\left(\begin{array}{cc}
e^{b t+b_{0}} & 0 \\
0 & e^{-b t-b_{0}}
\end{array}\right) .
$$


Remark. It follows from (7.52) and $\operatorname{tr} \omega=0$ that

$$
\operatorname{det} \varphi=\text { const . }
$$

Together with (7.36) this yields the identity

$$
A D+B C=\operatorname{const} \theta(V t+P) \theta[\varepsilon](V t+P)
$$

for $A, B, C, D$ given by (7.43). Therefore the polar divisors of $\ell_{i}(t), g_{i}(t)$ and of other coordinates of the top's frame are determined by the zeros of the theta functions $\theta(V t+P)$ and $\theta[\varepsilon](V t+P)$. This result agrees with the analysis in [9] and [10].

\subsection{Reduction to Two-Dimensional Theta Functions}

Since the Kowalewski flow on Jac $C$ is parallel to the Prym variety of the covering $C \rightarrow E$, it is desirable to express the dynamics entirely in terms of the theta functions related to this Prym variety (the elliptic theta function related to $E$ will enter through constant coefficients). The Prymian has polarization (1.2) and its period matrix is

$$
\Pi=\left(\begin{array}{cc}
2 \int_{b_{1}}\left(d u_{1}+d u_{3}\right) & \int_{b_{2}}\left(d u_{1}+d u_{3}\right) \\
2 \int_{b_{1}} d u_{2} & \int_{b_{2}} d u_{2}
\end{array}\right) .
$$

Let $\frac{1}{2} B_{0}$ be the period of $E$ :

$$
B_{0}=\int_{b_{1}}\left(d u_{1}-d u_{3}\right)
$$

We write the Prym vectors $V$ and $P$ entering in (7.42), and $C$ defined by (7.35), as

$$
V=\left(v_{1} / 2, v_{2}, v_{1} / 2\right), \quad P=\left(p_{1} / 2, p_{2}, p_{1} / 2\right), \quad C=\left(c_{1} / 2, c_{2}, c_{1} / 2\right),
$$

and denote

$$
w=\left(v_{1} t+p_{1}, v_{2} t+p_{2}\right), \quad c=\left(c_{1}, c_{2}\right) .
$$

We then have the following expressions for the theta functions occurring in (7.42):

$$
\begin{aligned}
\theta(V t+P)= & \left.\theta\left[\begin{array}{ll}
(0 & 0) \\
(0 & 0
\end{array}\right]\right)(w \mid \Pi) \theta\left[\begin{array}{l}
0 \\
0
\end{array}\right]\left(0 \mid B_{0}\right)+\theta\left[\begin{array}{cc}
(1 / 2 & 0) \\
(0 & 0)
\end{array}\right](w \mid \Pi) \theta\left[\begin{array}{c}
1 / 2 \\
0
\end{array}\right]\left(0 \mid B_{0}\right) \\
\theta(V t+P-R)= & \theta\left[\begin{array}{ll}
(0 & 0) \\
(0 & 0)
\end{array}\right](w \mid \Pi) \theta\left[\begin{array}{l}
0 \\
0
\end{array}\right]\left(2 r \mid B_{0}\right)+\theta\left[\begin{array}{cc}
(1 / 2 & 0) \\
(0 & 0)
\end{array}\right](w \mid \Pi) \theta\left[\begin{array}{c}
1 / 2 \\
0
\end{array}\right]\left(2 r \mid B_{0}\right), \\
\theta\left(V t+P+u\left(0_{ \pm}\right)\right)= & \theta\left[\begin{array}{ll}
(0 & 0) \\
(0 & 0
\end{array}\right](w \pm c \mid \Pi) \theta\left[\begin{array}{l}
0 \\
0
\end{array}\right]\left(r \mid B_{0}\right) \\
& +\theta\left[\begin{array}{cc}
(1 / 2 & 0) \\
(0 & 0)
\end{array}\right](w \pm c \mid \Pi) \theta\left[\begin{array}{c}
1 / 2 \\
0
\end{array}\right]\left(r \mid B_{0}\right) \\
\theta[\varepsilon](V t+P)= & \theta\left[\begin{array}{cc}
(0 & 0) \\
(0 & 1 / 2)
\end{array}\right](w \mid \Pi) \theta\left[\begin{array}{l}
0 \\
0
\end{array}\right]\left(0 \mid B_{0}\right) \\
& +\theta\left[\begin{array}{cc}
(1 / 2 & 0) \\
(0 & 1 / 2)
\end{array}\right](w \mid \Pi) \theta\left[\begin{array}{c}
1 / 2 \\
0
\end{array}\right]\left(0 \mid B_{0}\right)
\end{aligned}
$$




$$
\begin{aligned}
\theta[\varepsilon](V t+P-R)= & \theta\left[\begin{array}{cc}
(0 & 0) \\
(0 & 1 / 2
\end{array}\right](w \mid \Pi) \theta\left[\begin{array}{l}
0 \\
0
\end{array}\right]\left(2 r \mid B_{0}\right) \\
& +\theta\left[\begin{array}{cc}
(1 / 2 & 0) \\
(0 & 1 / 2)
\end{array}\right](w \mid \Pi) \theta\left[\begin{array}{c}
1 / 2 \\
0
\end{array}\right]\left(2 r \mid B_{0}\right), \\
\theta[\varepsilon]\left(V t+P+u\left(0_{ \pm}\right)\right)= & \theta\left[\begin{array}{cc}
(0 & 0) \\
(0 & 1 / 2)
\end{array}\right](w \pm c \mid \Pi) \theta\left[\begin{array}{l}
0 \\
0
\end{array}\right]\left(r \mid B_{0}\right) \\
& +\theta\left[\begin{array}{cc}
(1 / 2 & 0) \\
(0 & 1 / 2
\end{array}\right](w \pm c \mid \Pi) \theta\left[\begin{array}{c}
1 / 2 \\
0
\end{array}\right]\left(r \mid B_{0}\right) .
\end{aligned}
$$

Remark. Adding to $w$ a period vector of the form

$$
\left(\begin{array}{ll}
2 & 0 \\
0 & 1
\end{array} \mid \Pi\right)\left(\begin{array}{l}
M \\
N
\end{array}\right), \quad M, N \in \mathbb{Z}^{2},
$$

does not change the solutions (7.42). This shows that the mapping of the Liouville torus to Prym $C / E$ is one-to-one, as was already mentioned in Theorem 7.8.

\subsection{The Kowalewski-Dubrovin Equations}

In Sect. 4 the evolution of the line bundle $E_{L}$ of eigenvectors of $L(\lambda)$ was described in terms of the transition function $e^{t v}$. An alternative description in terms of the associated varying divisor is provided by the so-called Dubrovin equations. We shall derive these equations for the classical Kowalewski case. The divisor in question can be thought of as the divisor of zeros of any given component of the Baker-Akhiezer function, so let $\gamma_{1}(t), \gamma_{2}(t), \gamma_{3}(t)$ be the zeros of $\psi_{1}$. The evolution equations for $\gamma_{i}(t)$ are an immediate consequence of the fact that the flow linearizes on the Jacobian. From (7.20) we have

$$
\sum_{i} u\left(\gamma_{i}(t)\right)=V t+D+K
$$

where $K$ is Riemann's constant. Hence

$$
\sum_{i} \frac{d}{d t} u\left(\gamma_{i}(t)\right)=V
$$

To write this more explicitly we choose a basis of holomorphic differentials on $C$ [recall that $C$ is defined by $(5.3)]$ :

$$
\begin{gathered}
d v=\frac{d z}{z\left(\mu^{2}-d_{1}(z)\right)}, \\
d w_{1}=\frac{d z}{\mu z\left(\mu^{2}-d_{1}(z)\right)}, \\
d w_{2}=\left(\frac{1}{\mu z}+\frac{2}{\mu\left(\mu^{2}-d_{1}(z)\right)}\right) d z .
\end{gathered}
$$

Here $d v$ is a differential on $E$, and $d w_{i}$ are Prym differentials. Recalling the expression (4.10) for $V$ (equivalently, $d u(p)=(V+o(1)) d k$ as $p \rightarrow \infty_{+}$, where 
$k=1 / \sqrt{z}$ is a local parameter at $\infty_{+}$) we have

$$
\begin{gathered}
\sum_{i} \frac{d z_{i} / d t}{z_{i}\left(\mu_{i}^{2}-d_{1}\left(z_{i}\right)\right)}=0, \\
\sum_{i} \frac{d z_{i} / d t}{\mu_{i} z_{i}\left(\mu_{i}^{2}-d_{1}\left(z_{i}\right)\right)}=0, \\
\sum_{i}\left(\frac{1}{\mu_{i} z_{i}}+\frac{2}{\mu_{i}\left(\mu_{i}^{2}-d_{1}\left(z_{i}\right)\right)}\right) \frac{d z_{i}}{d t}=-2,
\end{gathered}
$$

where $z_{i}=z\left(\gamma_{i}\right)$ and $\mu_{i}=\mu\left(\gamma_{i}\right)$.

It remains an interesting problem to determine the Hamiltonian structure for (7.69) and to use these equations to compute the action-angle variables of the Kowalewski top following the pattern indicated by Novikov and Veselov [12].

\subsection{Motion of the Kowalewski Top in the Case $(\ell, g)=0$}

If $(\ell, g)=0$, the curve $E$ degenerates into a rational curve which is a two-sheeted cover of the $z$-plane,

$$
\left(y-d_{1}(z)\right)^{2}=4\left(z^{2}-2 H z+1+H^{2}-1 / 4 I_{2}\right) .
$$

The curve $C$ is given by the equation $\mu^{2}=y$, or

$$
\left(\mu^{2}-d_{1}(z)\right)^{2}=4\left(z^{2}-2 H z+1+H^{2}-1 / 4 I_{2}\right)
$$

and has genus 2 . The covering $C \rightarrow E$ has 6 ramification points $\infty_{ \pm}, 0_{ \pm}, p_{1}, p_{2}$ (we use the same notation as in Sect. $\left.5: z\left(\infty_{ \pm}\right)=\infty, z\left(0_{ \pm}\right)=0, y\left(\infty_{-}\right)=0, y\left(\infty_{+}\right)=\infty\right)$. The flip of sign $\mu \mapsto-\mu$ defines a hyperelliptic involution on $C$. In the variables $u=\sqrt{2} \mu z x, x=1 / 2\left(\mu^{2}-1 / z\right)$, Eq. (7.71) takes on the usual hyperelliptic form:

$$
u^{2}=x\left(x^{2}+2 H x+1 / 4 I_{2}\right)\left(x^{2}+2 H x+1 / 4 I_{2}-1\right) .
$$

Notice that it is different from the Kowalewski curve which for $(\ell, g)=0$ is given by

$$
u^{2}=x\left((x-H)^{2}-1 / 4 I_{2}\right)\left((x-H)^{2}-1 / 4 I_{2}+1\right) .
$$

The fact that there are various hyperelliptic curves associated with the Kowalewski top which are different from the classical Kowalewski curve was pointed out in [10]. The motion of the top linearizes on the Jacobians of the curves (7.72), (7.73) which are isogeneous to one another.

Let us regard the curve $C$ defined by (7.72) as a two-sheeted covering of the $x$-plane. The fixed points of the hyperelliptic involution $u \mapsto-u$ are $\infty_{ \pm}, 0_{ \pm}, p_{1}, p_{2}$. We choose a canonical basis of cycles on $C$ as shown on Fig. 2.

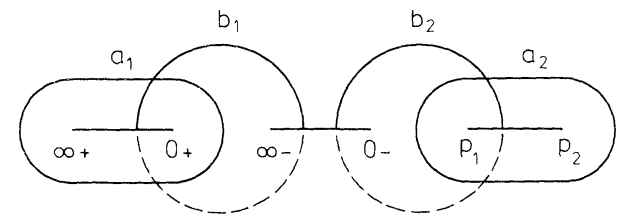

Fig. 2. Represents the curve $C$ in the case $(\ell, g)=0$ as two copies of the $x$-plane glued along the cuts $\left[\infty_{+}, 0_{+}\right],\left[0_{-}, \infty_{-}\right]$and $\left[p_{1}, p_{2}\right]$. Continuous lines show (parts of) the cycles on the upper sheet, dotted lines refer to the lower sheet 
As usual, we define the period matrix with respect to this basis. Let $\theta\left[\begin{array}{l}\alpha \\ \beta\end{array}\right]$ be the associated Riemann's theta function with characteristics. Then the solutions of the equations of motion are given by

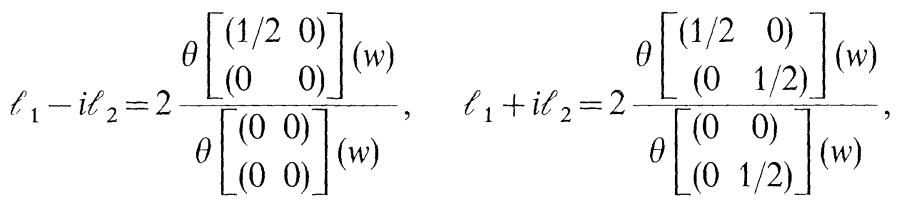

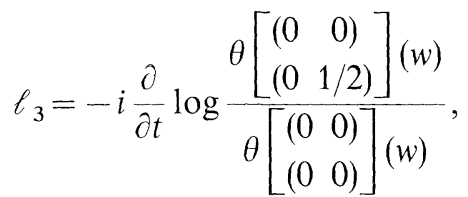

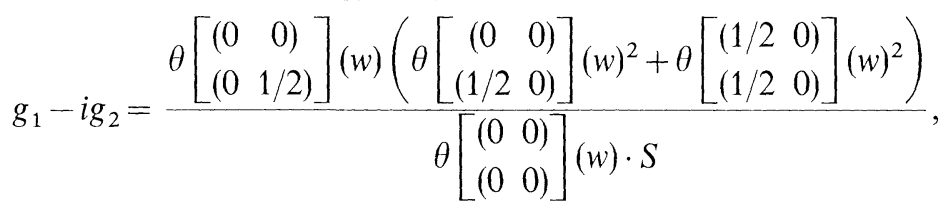

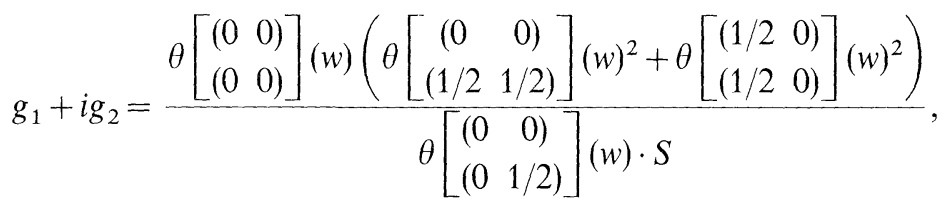

$$
\begin{aligned}
& g_{3}=\frac{i}{S}\left(\theta\left[\begin{array}{cc}
(0 & 0) \\
(1 / 2 & 0)
\end{array}\right](w) \theta\left[\begin{array}{cc}
(1 / 2 & 0) \\
(1 / 2 & 1 / 2)
\end{array}\right](w)\right.
\end{aligned}
$$

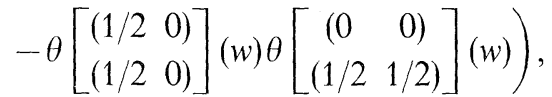

where

$$
\left.S=\theta\left[\begin{array}{cc}
(0 & 0) \\
(1 / 2 & 0)
\end{array}\right](w) \theta\left[\begin{array}{cc}
(0 & 0) \\
(1 / 2 & 1 / 2)
\end{array}\right](w)+\theta\left[\begin{array}{cc}
(1 / 2 & 0) \\
(1 / 2 & 0)
\end{array}\right](w) \theta\left[\begin{array}{cc}
(1 / 2 & 0) \\
(1 / 2 & 1 / 2
\end{array}\right]\right)(w) .
$$

Here $w=V t+D$ and $V$ is the velocity vector of the Kowalewski flow on Jac $C$ given by (4.10). Formulae (7.74) imply that the (compactified) Liouville torus is in the present case an unramified double covering of Jac $C$. Its polarization is again (1.2). This is in agreement with the qualitative analysis in [10].

To conclude, we point out the following curious fact: by deleting the first row and column in (6.3) one gets the L-matrix of a Lax pair for the GoryachevChaplygin top, which leads to new formulae for its solutions [211.

Acknowledgement. The authors wish to thank Prof. L. D. Faddeev for valuable encouragement.

\section{References}

1. Kowalewski, S.: Sur le problème de la rotation d'un corps solide autour d'un point fixe. Acta Math. 12, 177-232 (1889)

2. Kötter, F.: Sur le cas traité par Mme Kowalewski de rotation d'un corps solide autour d'un point fixe. Acta Math. 17, 209-264 (1893) 
3. Dubrovin, B.A., Krichever, J.M., Novikov, S.P.: Integrable systems. I. In: Modern problems in mathematics. Fundamental developments. Vol. 4 (Dynamical systems 4) Itogi Nauki i Tekhniki. Moscow: VINITI 1985, pp. 179-285

4. Perelomov, A.M.: Lax representations for the systems of S. Kowalewskaya type. Commun. Math. Phys. 81, 239-243 (1981)

5. Reyman, A.G., Semenov-Tian-Shansky, M.A.: Lax representation with a spectral parameter for the Kowalewski top and its generalizations. Lett. Math. Phys. 14, 55-62 (1987)

6. Reyman, A.G., Semenov-Tian-Shansky, M.A.: Reduction of Hamiltonian systems, affine Lie algebras and Lax equations I, II. Invent. Math. 54, 81-100 (1979); 63, 423-432 (1981)

7. Reyman, A.G., Semenov-Tian-Shansky, M.A.: A new integrable case of the motion of the 4-dimensional rigid body. Commun. Math. Phys. 105, 461-472 (1986)

8. Haine, L., Horozov, E.: A Lax pair for Lowalewski's top. Physica 29 D, 173-180 (1987)

9. Adler, M., van Moerbeke, P.: The Kowalewski and Hénon-Heiles motions as Manakov geodesic flows on SO(4) - A two-dimensional family of Lax pairs. Commun. Math. Phys. 113, $659-700(1988)$

10. Horozov, E., van Moerbeke, P.: Abelian surfaces of polarization (1.2) and Kowalewski's top. Commun. Pure Appl. Math. (to appear) 1988

11. Dubrovin, B.A., Matveev, V.B., Novikov, S.P.: Nonlinear equations of KdV type, finite-zone linear operators and abelian varieties. Usp. Mat. Nauk 31, 55-136 (1976) [Russ. Math. Surv. 31, 59-146 (1976)]

12. Veselov, A.P., Novikov, S.P.: Poisson brakcets and complex tori. Trudy Mos. Mat. O.-va 165, 49-61 (1984)

13. Bobenko, A.I.: Solutions of the classical integrable tops via the inverse scattering method. LOMI Preprint P-10-87, Leningrad 1987

14. Bogoyavlensky, O.I.: Euler equations on finite-dimensional Lie algebras arising in physical problems. Commun. Math. Phys. 95, 307-315 (1984)

15. Komarov, I.V.: A generalization of the Kowalewski top. Phys. Lett. 123 A, 14-15 (1987)

16. Reyman, A.G., Semenov-Tian-Shansky, M.A.: Group-theoretical methods in the theory of integrable systems. In: Modern Problems in Mathematics. Fundamental Developments, Vol. 16 (Dynamical systems 7) Itogi Nauki i Tekhniki. Moscow: VINITI 1987, pp. 119-195

17. Reyman, A.G.: Integrable Hamiltonian systems connected with graded Lie algebras. In: Differential geometry. Lie groups and mechanics II. Zap. Nauchn. Semin. LOMI 95, 3-54 (1980) [J. Sovict Math. 19, 1507-1545 (1982)]

18. Guillemin, V., Sternberg, S.: The moment map and collective motion. Ann. Phys. 127, 220-253 (1980)

19. Griffiths, P., Harris, J.: Principles of algebraic geometry. New York: Wiley 1978

20. Fay, J.D.: Theta functions on Riemann surfaces. Lecture Notes in Mathematics, vol. 352. Berlin, Heidelberg, New York: Springer 1973

21. Bobenko, A.I., Kuznetsov, V.B.: Lax representation for the Goryachev-Chaplygin top and new formulae for its solutions. J. Phys. A 21, 1999-2006 (1988)

22. Reyman, A.G., Semenov-Tian-Shansky, M.A.: Lax representation with a spectral parameter for the Kowalewski top and its generalizations. In: Plasma theory and nonlinear and turbulent processes in physics (Proceedings of the $1987 \mathrm{Kiev}$ conference), pp. 135-152. Singapore: World Scientific 1988

23. Reyman, A.G.: New results on the Kowalewski top. In: Nonlinear Evolutions (Proceedings of the IV Workshop on Nonlinear Evolution Equations and Dynamical Systems, Balaruc-lesBains, 1987). Singapore: World Scientific 1988

24. Yehia H.: New integrable cases of the motion of a gyrostat. Vestn. Mosk. Univ., Ser. 1, No. 4, 88-90 (1987)

Communicated by Ya. G. Sinai

Received February 15, 1988; in revised form September 22, 1988 